\title{
Biofeedback Systems for Gait Rehabilitation of Individuals with Lower-Limb Amputation: A Systematic Review
}

\author{
Rafael Escamilla-Nunez ${ }^{1,2}$, Alexandria Michelini ${ }^{1,2}$ (D) and Jan Andrysek 1,2,*(D) \\ 1 Institute of Biomaterials and Biomedical Engineering, University of Toronto, Toronto, ON M4Y 1R5, Canada; \\ rafael.escamilla@mail.utoronto.ca (R.E.-N.); a.michelini@mail.utoronto.ca (A.M.) \\ 2 Bloorview Research Institute, Holland Bloorview Kids Rehabilitation Hospital, Toronto, \\ ON M4G 1R8, Canada \\ * Correspondence: jandrysek@hollandbloorview.ca
}

Received: 18 February 2020; Accepted: 12 March 2020; Published: 14 March 2020

check for updates

\begin{abstract}
Individuals with lower-limb amputation often have gait deficits and diminished mobility function. Biofeedback systems have the potential to improve gait rehabilitation outcomes. Research on biofeedback has steadily increased in recent decades, representing the growing interest toward this topic. This systematic review highlights the methodological designs, main technical and clinical challenges, and evidence relating to the effectiveness of biofeedback systems for gait rehabilitation. This review provides insights for developing an effective, robust, and user-friendly wearable biofeedback system. The literature search was conducted on six databases and 31 full-text articles were included in this review. Most studies found biofeedback to be effective in improving gait. Biofeedback was most commonly concurrently provided and related to limb loading and symmetry ratios for stance or step time. Visual feedback was the most used modality, followed by auditory and haptic. Biofeedback must not be obtrusive and ideally provide a level of enjoyment to the user. Biofeedback appears to be most effective during the early stages of rehabilitation but presents some usability challenges when applied to the elderly. More research is needed on younger populations and higher amputation levels, understanding retention as well as the relationship between training intensity and performance.
\end{abstract}

Keywords: amputee; biofeedback; gait; locomotion; lower-limb amputation; prosthesis; real-time feedback; sensory feedback; rehabilitation; wearable systems

\section{Introduction}

Lower-limb amputation (LLA) is associated with major rehabilitation challenges and lifelong mobility limitations. Limb loss not only hinders aspects of motor control, but it also reduces the sensory feedback information and proprioception that are associated with the peripheral nervous system [1-4]. As a result, individuals with LLA often walk slower and expend more energy than non-amputees [5]. They also exhibit atypical gait and loading patterns [5-7] that may be associated with long-term secondary health issues including chronic back pain and joint problems [8]. Moreover, poor balance and gait function in individuals with LLA can lead to the fear of falling and a greater incidence of falls [9-12], with more than half of ambulating adults with LLA falling at least once per year [11]. The consequences of these falls include injury and hospitalization [12,13], heightened fear of falling leading to prosthesis disuse [14,15], and the subsequent social withdrawal reducing their ability to recover from the trauma, both physically and psychologically $[14,16]$.

Improving balance and gait is an important part of the rehabilitation process. Gait retraining, which is typically provided by a physiotherapist or prosthetist, includes the observation of gait 
deviations or atypical movement patterns and the provision of verbal cuing to promote corrections. However, these conventional methods have several limitations. The detection of gait deviations is limited to a subjective assessment of gross movement patterns [17]. Further, gait training sessions are limited in duration and frequency. Many health care systems are under-resourced despite the need for rehabilitation, making it challenging for clinics and rehabilitation hospitals to give adequate levels of therapy services [18]. Many patients face serious barriers (e.g., long travel times, school/work absences, etc.) accessing services, and, at best, only undertake unsupervised lower limb exercises at home [19-21].

Technology-driven approaches, such as virtual reality, therapy-focused videogames, and biofeedback (BFB) systems, are promising modalities for augmenting rehabilitation in the clinical facility and at home [22-25]. These technologies take advantage of motor learning and relearning strategies to accelerate the gait rehabilitation learning process. A major benefit of BFB systems, especially wearable systems, is the ability to provide real-time, continuous feedback to reinforce physiotherapy/prosthetist (PT) goals and good gait habits [26-29]. BFB approaches in rehabilitation consist of utilizing external sensors, such as inertial measurement units, goniometers, pressure sensors, force plates, and motion capture systems, to measure specific parameters relating to postural balance, gait kinetics, and kinematics [22,30-33]. Subsequently, real-time biomechanical information, which is captured by the external sensors, is communicated to the BFB users to alter their performance through some appropriate feedback modality (i.e., visual [34,35], auditory [36], haptic [25,26,37], or multimodal feedback $[38,39])$.

BFB approaches in rehabilitation have been studied in a variety of patient populations including stroke [37,40-42], Parkinson's disease [43-45], cerebral palsy [46], vestibular deficits [47,48], diabetes [49], and upper-limb [50,51] and lower-limb amputees [31,52,53]. As well as in a variety of applications, including static and dynamic postural balance [54-56], walking [57-59], stairs management [60], obstacle avoidance [61], floor conditions identification [62], and sensory perception [31,53,63], to mention a few. In 2018, a mapping review [64] was published regarding the use of BFB for gait retraining. The review covered a variety of patient populations across 173 reviewed articles. The results showed that, during walking, the most common feedback modality, feedback gait parameter, and external sensory configuration were visual feedback, kinematic gait parameters, and pressure sensors fixed to the feet or on the feet insoles, respectively. In addition, it revealed that most of the studies (approximately 90\%), tested BFB in a laboratory setting, and more than a half (i.e., 53\%) of all studies performed a single intervention session. The relevance and extent of applicability to individuals with LLA remains uncertain despite these valuable new insights into BFB systems, since LLA was the subject of only eight of the 173 reviewed articles.

Similarly, earlier reviews relating to BFB have not directly focused on individuals with LLA, but more generally on a range of diagnoses. A 2010 systematic review [65] investigated the application of BFB in older adults with balance and mobility disorders [65]. Most of the reviewed articles involved post-stroke participants or community-dwelling elderly subjects. The three articles dealing with LLA showed that participants were able to adjust their weight on the injured leg during walking $[66,67]$ and to improve sway and weight distribution during standing [68] when auditory feedback was provided about weight-bearing. Another systematic review [22] published in 2010 investigated the effectiveness of BFB systems for gait retraining of several related pathologies (e.g., cerebrovascular accident, hip fracture, etc.), including only one study on LLA. This review showed the potential of BFB systems to produce moderate to large short-term treatment effects and improvements as compared to conventional therapies when providing biomechanical information to BFB users [22]. It was further concluded that kinematic and spatiotemporal feedback gait parameters were the most targeted training interventions amongst stroke patients, while the kinetic feedback parameters were of primary interest for LLA [22].

While substantial research has been conducted toward the establishment of prosthetic BFB, to the authors' knowledge, no study has attempted to consolidate this information to provide a comprehensive 
assessment of the state of the science, and the potential role of BFB in the treatment of LLA. In this regard, the main aim of the systematic review was to provide insights and recommendations towards the development of an effective, robust, and user-friendly wearable BFB system that can be integrated into existing prosthetic systems to assist individuals with LLA during the gait rehabilitation process. Specifically, this systematic review aimed to identify: (1) targeted populations and demographics within prosthetics, (2) targeted gait and biomechanical parameters, (3) the most common BFB system designs, including the measured biomechanical signals and feedback modalities, (4) the main technical and clinical limitations of current BFB systems, (5) main clinical evidence relating to BFB efficacy and effectiveness, and (6) future directions and applications of BFB systems for individuals with LLA.

\section{Methods}

The systematic review was conducted following the Preferred Reporting Items for Systematic Reviews and Meta-Analyses (PRISMA), see Supplementary Materials. The review protocol is register to PROSPERO, which is an international prospective register of systematic reviews (Ref: CRD42020142222).

\subsection{Search Strategy}

A literature search was performed on October 2019 on the following databases: Medline (1946-2019), Embase (1947-2019), PubMed (1971-2019), IEEE (1872-2019), Web of Science (1900-2019), and Scopus (2004-2019). These six databases were chosen as they cover most of the literature in the fields of engineering and medicine. Keywords "biofeedback", "amputee", and "gait" were matched with MeSH (medical subject headings) terms and subheadings when relevant. Truncations and wildcards were utilized to capture all forms of a root word. Table 1 shows an example of a search strategy performed in one of the databases (i.e., Medline). Minor modifications of the keyword terms were used in the different databases to expand the search results.

Table 1. Keyword search strategy employed in Medline database.

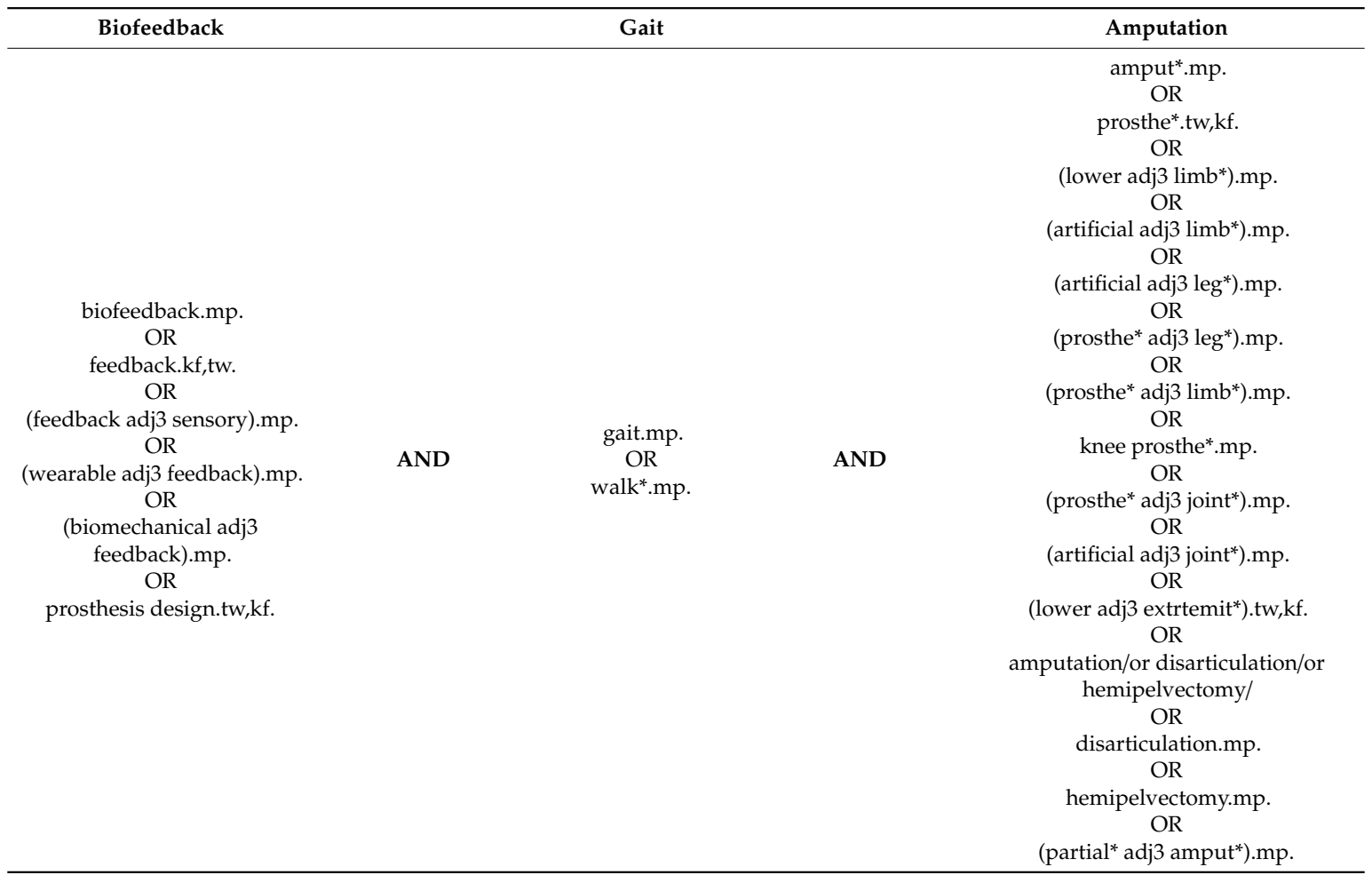

kf: keyword heading word; tw: text word; mp: multi-purpose field search (Title, Original Title, Abstract, and Subject Heading, among others depending on the database). 


\subsection{Inclusion and Exclusion Criteria}

The article inclusion/exclusion criteria were divided into three main sections: (i) study population, (ii) biofeedback application, and (iii) publication type (Table 2).

Table 2. Framework for Inclusion/Exclusion of Eligible Studies.

\begin{tabular}{|c|c|c|}
\hline \multirow{2}{*}{ Section } & \multicolumn{2}{|r|}{ Criteria } \\
\hline & Inclusion & Exclusion \\
\hline Study Population & $\begin{array}{l}\text { - Individuals with } \\
\text { lower-limb amputation } \\
\text { Able-bodied subjects with explicit } \\
\text { future application to lower-limb } \\
\text { amputation population }\end{array}$ & $\begin{array}{l}\text { - Individuals with upper-limb amputation } \\
\text { - Other populations }\end{array}$ \\
\hline Biofeedback application & - Gait or walking applications & $\begin{array}{l}\text { - } \quad \text { BFB applied to any activity different from } \\
\text { walking (e.g., balance, running, golfing) } \\
\text { BFB applied for robotic assistance (e.g., } \\
\text { exoskeletons) or computer simulations (e.g., } \\
\text { virtual human models or virtual prostheses) } \\
\text { - } \quad \text { BFB used exclusively to assess user sensory } \\
\text { perception of participants without targeting a } \\
\text { gait parameter while walking } \\
\text { BFB designs not clinically tested or mainly used } \\
\text { for gait event detection or system validation } \\
\text { BFB does not explicitly inform the user of errors } \\
\text { that they are exhibiting (e.g., mirror therapy } \\
\text { studies, studies where users observe videos of } \\
\text { their own gait patterns without having } \\
\text { deviations pointed out to the them) }\end{array}$ \\
\hline Publication Type & $\begin{array}{ll}\text { - } & \text { Peer reviewed journal articles } \\
\text { - } & \text { Peer review conference proceedings } \\
\text { - } & \text { Studies published in English } \\
\text { - } & \text { Full-text articles }\end{array}$ & $\begin{array}{ll}-\quad & \text { Literature reviews } \\
-\quad & \text { Survey studies }\end{array}$ \\
\hline
\end{tabular}

\subsection{Screening and Data Extraction}

After the duplicates were removed, two independent reviewers screened the titles and abstracts of retrieved studies for relevance using the predefined eligibility criteria (Table 2) (R.E. \& A.M.). The remaining studies received full-text assessments. Articles with titles and abstracts that did not provide enough information for the article screening process were fully reviewed. The data were extracted based on the study aims. Accordingly, the following aspects for data extraction were considered: (i) year of publication; (ii) BFB objective and application; (iii) characteristics of the sample population; (iv) BFB design (BFB modality, BFB device, feedback strategy, sensors/transducers); (v) testing conditions (clinical/laboratory settings or field-based studies and treadmill or overground walking); (vi) outcome measures (targeted gait parameters, physical, physiological, or any other parameters, including questionnaires); (vii) experimental protocol (information related to subject's testing, such as number of sessions, duration, frequency, number of trials, follow-up interventions); and, (viii) key findings that were related to the efficacy and effectiveness of current BFB systems as gait rehabilitation tools for individuals with LLA. A third reviewer (J.A.) resolved the ambiguities or disagreements in the independent reviews of the articles (reviewers R.E. \& A.M.). Additionally, the references of all included articles were scanned to identify other relevant studies that were missed in the original search.

\subsection{Risk of Bias (Quality) Assessment}

Most of the inclusions were peer-reviewed journal articles thus maintaining the quality of this systematic review and reducing the risk of publication bias. Two independent reviewers assessed all articles that met the inclusion criteria (R.E. \& A.M.). In addition, a quality assessment was performed using a customized data extraction formula (Table 3). The approach was based on previous 
standardized methods [69-71] and reviews, and allows for data extraction that is relevant to the topic of interest [72,73]. For instance, Peters et al. [72] assessed 20 reviewed articles by using 19 appraisal questions as quality indicators. The appraisal questions were designed to collect information regarding the main research aims. Similarly, Ku et al. [73] utilized 14 appraisal questions to evaluate 23 articles that were related to balance control of individuals with LLA during quiet standing. The evaluation process in the current systematic review was adapted from these previously established appraisal criteria (Table 3) [69-73]. Accordingly, the score of each article provided a standardized measure for assessing the quality of research among the articles. Reviewers R.E. \& A.M. independently applied the ratings and reviewer J.A. resolved disagreement.

Table 3. Criteria for Quality Assessment ${ }^{* *}$.

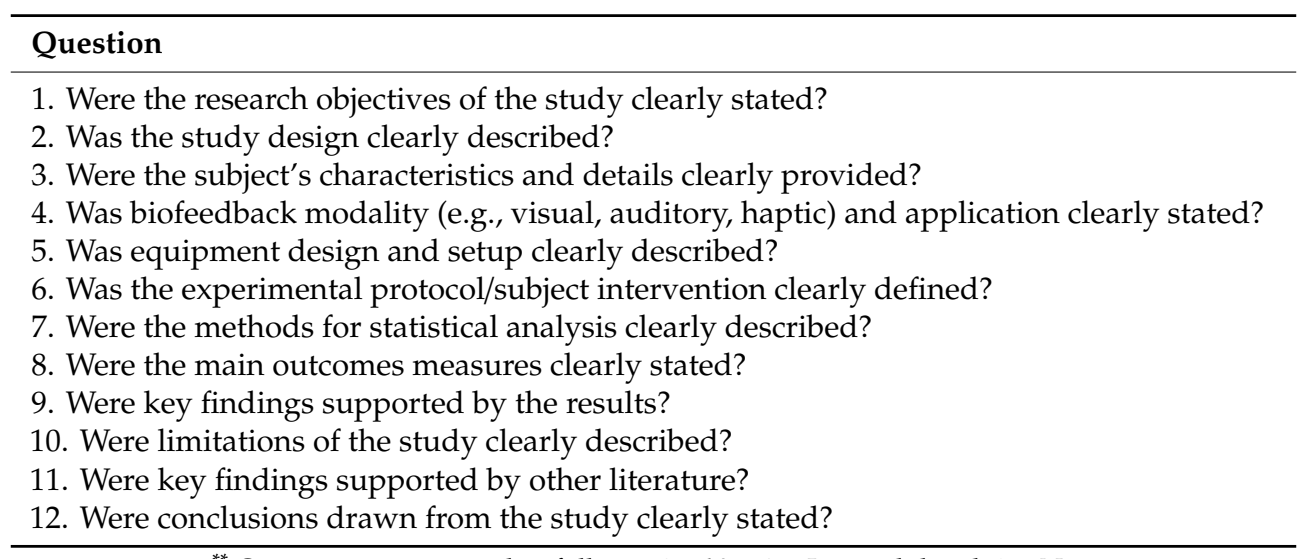

${ }^{* *}$ Questions were scored as follows: $2=$ Yes; $1=$ Limited detail; $0=$ No.

\section{Results}

\subsection{Search Results}

The initial search yielded 2456 studies (i.e., Medline 440, PubMed 426, Embase 550, IEEE 221, Web of Science 281 and Scopus 538). After the duplicates were removed, the title and abstract of 1419 articles were screened for potential relevance. Seventy-two $(n=72)$ full-text articles were assessed for eligibility. Following the application of the eligibility criteria, thirty-one $(n=31)$ full-text studies were included in this systematic review. The flow diagram summarizes the overall review process (Figure 1). The most common reasons for the exclusion of articles during full-text assessment included: (1) BFB systems not being tested on individuals with LLA, (2) mainly used for gait event detection, and (3) used to assess user's sensory perception (i.e., reaction time and subject's accuracy in response to stimulation). 


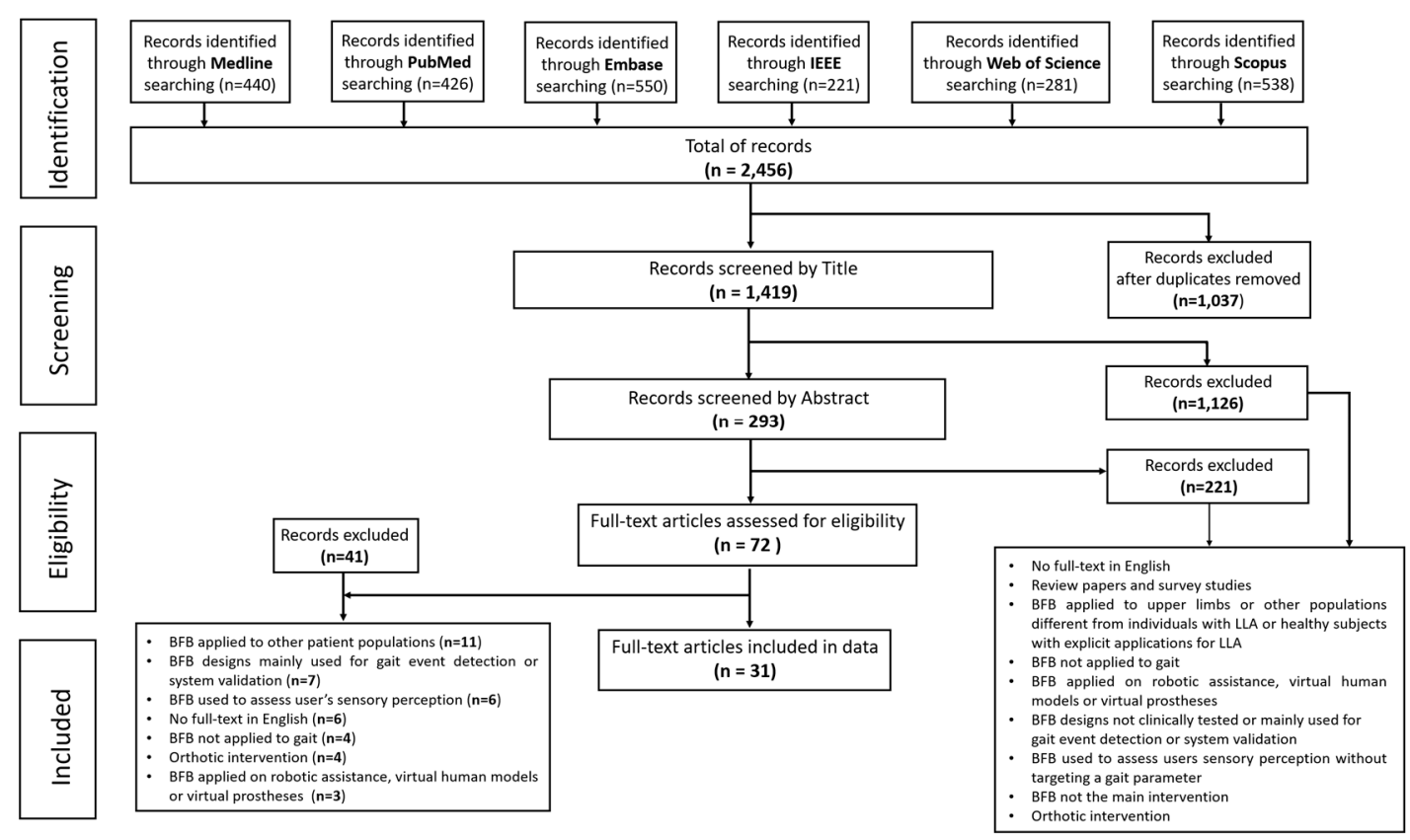

Figure 1. Preferred Reporting Items for Systematic Reviews and Meta-Analyses (PRISMA) flow diagram of the screening and data extraction process.

\subsection{Quality of Reviewed Articles}

Table 4 depicts the results of the criteria applied to assess the quality of the reviewed articles. Most articles were high quality and included complete information about the research objectives, study design, participants characteristics, BFB modality and application, BFB components, primary gait outcome measures, key findings supported by results, and conclusions. However, some articles presented limited information about experimental protocol and study limitations. Whereas, other studies presented limited or null information about statistical analyses and key findings supported by other literature. The results showed that 16 out of the 31 studies satisfied at least $85 \%$ of the criteria. Nine studies ranged from $70 \%$ to $85 \%$, and six studies scored less than $70 \%$.

It should be noted that Lee et al. published five articles with similar methodology, data sets, and outcomes between the years of 2007-2013 [56,74-77].

\subsection{Key Data Extracted from Reviewed Articles}

BFB systems applied as a gait rehabilitation tool for prosthetic users has gained popularity over recent years, with most articles published from 2007 to $2019(n=21,68 \%)$. The participants with transtibial amputation were most studied $(n=17,55 \%)$ and the included studies had a median sample size of seven participants. Only two studies $[34,66]$ compared BFB performance versus a control group of healthy subjects. Most of the studies included middle-aged (aged 30-59 years) and elderly (above 59 years) prosthetic users. Study participants had prosthetic experience ranging from one month to 53 years.

Only six studies presented detailed characteristics about the prosthetic components (i.e., prosthetic joint, foot, and socket) utilized to evaluate BFB [34,59,78-81]. During BFB testing, most of the participants wore their prescribed prosthesis (i.e., passive mechanical or microprocessor-controlled knee prostheses). In terms of BFB effectiveness and prosthetic components, the results showed that BFB systems were capable of improving the gait performance of individuals with LLA, regardless of the type of prosthetic components (i.e., passive mechanical knee or microprocessor-controlled knee or powered knee prostheses) [35,59,79-82]. 
Table 4. Quality analysis results from reviewed articles.

\begin{tabular}{|c|c|c|c|c|c|c|c|c|c|c|c|c|c|c|c|}
\hline \multirow{2}{*}{ Study, Author } & \multirow{2}{*}{ Year } & \multicolumn{12}{|c|}{ Question } & \multirow{2}{*}{ Total Score } & \multirow{2}{*}{ Overall Percentage } \\
\hline & & 1 & 2 & 3 & 4 & 5 & 6 & 7 & 8 & 9 & 10 & 11 & 12 & & \\
\hline [59] Petrini et al. & 2019 & 1 & 2 & 2 & 2 & 2 & 2 & 2 & 2 & 2 & 2 & 2 & 1 & $22 / 24$ & 92 \\
\hline [57] Fiedler et al. & 2019 & 2 & 2 & 2 & 2 & 2 & 2 & 0 & 1 & 2 & 2 & 1 & 2 & $23 / 24$ & 96 \\
\hline [80] Petrini et al. & 2019 & 2 & 2 & 1 & 2 & 2 & 2 & 2 & 2 & 2 & 2 & 2 & 1 & $22 / 24$ & 92 \\
\hline [35] Brandt et al. & 2019 & 2 & 2 & 2 & 2 & 2 & 1 & 2 & 2 & 2 & 2 & 2 & 2 & $23 / 24$ & 96 \\
\hline [86] Dietrich et al. & 2018 & 2 & 2 & 2 & 2 & 2 & 1 & 2 & 2 & 2 & 2 & 2 & 2 & $23 / 24$ & 96 \\
\hline [34] Esposito et al. & 2017 & 2 & 2 & 1 & 2 & 2 & 2 & 2 & 2 & 2 & 2 & 2 & 2 & $23 / 24$ & 96 \\
\hline [87] Maldonado et al. & 2017 & 2 & 2 & 1 & 2 & 2 & 2 & 1 & 2 & 2 & 1 & 0 & 2 & $19 / 24$ & 79 \\
\hline [58] Crea et al. & 2017 & 2 & 2 & 1 & 2 & 2 & 2 & 2 & 2 & 2 & 2 & 2 & 2 & $23 / 24$ & 96 \\
\hline [28] Plauche et al. & 2016 & 2 & 2 & 2 & 2 & 2 & 2 & 0 & 2 & 2 & 2 & 2 & 1 & $21 / 24$ & 88 \\
\hline [84] Pagel et al. & 2016 & 2 & 2 & 2 & 2 & 2 & 1 & 2 & 2 & 2 & 2 & 2 & 2 & $23 / 24$ & 96 \\
\hline [82] Huang et al. & 2016 & 2 & 2 & 2 & 2 & 2 & 2 & 2 & 2 & 2 & 2 & 1 & 2 & $23 / 24$ & 96 \\
\hline [30] Crea et al. & 2015 & 2 & 2 & 1 & 2 & 2 & 2 & 2 & 2 & 2 & 2 & 2 & 2 & $23 / 24$ & 96 \\
\hline [77] Lee et al. & 2013 & 2 & 2 & 2 & 2 & 2 & 1 & 0 & 2 & 1 & 1 & 1 & 2 & $18 / 24$ & 75 \\
\hline [88] Redd et al. & 2012 & 2 & 2 & 1 & 2 & 2 & 2 & 2 & 2 & 2 & 2 & 2 & 2 & $23 / 24$ & 96 \\
\hline [36] Yang et al. & 2012 & 2 & 2 & 2 & 2 & 2 & 1 & 1 & 2 & 2 & 2 & 0 & 2 & $20 / 24$ & 83 \\
\hline [81] Darter et al. & 2011 & 2 & 2 & 2 & 2 & 2 & 2 & 0 & 2 & 2 & 2 & 2 & 2 & $22 / 24$ & 92 \\
\hline [76] Lee et al. & 2010 & 2 & 2 & 2 & 2 & 2 & 1 & 0 & 2 & 1 & 1 & 1 & 2 & $18 / 24$ & 75 \\
\hline [75] Lee et al. & 2009 & 2 & 2 & 2 & 2 & 2 & 1 & 0 & 2 & 1 & 1 & 1 & 2 & $18 / 24$ & 75 \\
\hline [74] Lee et al. & 2008 & 2 & 2 & 2 & 2 & 2 & 1 & 0 & 2 & 1 & 1 & 1 & 2 & $18 / 24$ & 75 \\
\hline [56] Lee et al. & 2007 & 2 & 2 & 2 & 2 & 2 & 1 & 0 & 2 & 1 & 1 & 1 & 2 & $18 / 24$ & 75 \\
\hline [66] Isakov et al. & 2007 & 2 & 2 & 2 & 1 & 2 & 2 & 2 & 2 & 2 & 0 & 0 & 2 & $19 / 24$ & 79 \\
\hline [89] Davis et al. & 2004 & 2 & 2 & 2 & 1 & 1 & 2 & 2 & 2 & 2 & 2 & 2 & 2 & $22 / 24$ & 92 \\
\hline [90] Chow et al. & 2000 & 2 & 2 & 2 & 2 & 2 & 2 & 2 & 2 & 2 & 1 & 1 & 2 & $22 / 24$ & 92 \\
\hline [91] Dingwell et al. & 1996 & 2 & 2 & 1 & 2 & 2 & 2 & 2 & 2 & 2 & 2 & 2 & 2 & $23 / 24$ & 96 \\
\hline [83] Sabolich et al. & 1994 & 2 & 2 & 2 & 2 & 2 & 2 & 1 & 2 & 2 & 1 & 0 & 2 & $20 / 24$ & 83 \\
\hline [17] Flowers et al. & 1986 & 2 & 1 & 1 & 2 & 2 & 0 & 0 & 1 & 2 & 2 & 0 & 2 & $15 / 24$ & 63 \\
\hline [92] Clippinger et al. & 1982 & 1 & 1 & 2 & 2 & 2 & 2 & 0 & 1 & 1 & 0 & 1 & 1 & $14 / 24$ & 58 \\
\hline [93] Gapsis et al. & 1982 & 2 & 1 & 2 & 2 & 2 & 1 & 1 & 1 & 1 & 1 & 1 & 1 & $16 / 24$ & 67 \\
\hline [85] Fernie et al. & 1978 & 1 & 1 & 1 & 1 & 1 & 1 & 0 & 1 & 1 & 1 & 0 & 1 & $10 / 24$ & 42 \\
\hline [94] Zimnicki et al. & 1976 & 1 & 1 & 1 & 2 & 2 & 2 & 0 & 1 & 1 & 1 & 0 & 1 & $13 / 24$ & 54 \\
\hline [95] Warren et al. & 1975 & 2 & 1 & 1 & 1 & 1 & 2 & 0 & 1 & 1 & 1 & 1 & 1 & $13 / 24$ & 54 \\
\hline
\end{tabular}

Questions were scored as follows: $2=$ Yes; 1 = Limited detail; $0=$ No. Questions were related to the description or justification of (1) Objectives; (2) Study Design; (3) Participant characteristics; (4 and 5) BFB system; (6) Experimental protocol; (7) Statistics; (8) Main Outcome Measures; (9 \& 11) Key Findings; (10) Limitations; and, (12) Conclusions.

FSRs (force sensitive resistors) sensors that were attached to the plantar surface of the prosthetic foot were the most frequently used transducer for measuring the targeted gait parameters. The most commonly targeted gait parameters were related to limb loading, ground reaction forces, and symmetry ratios for stance or step time. Visual feedback was the most used modality, followed by auditory and haptic. Haptic feedback has been most frequently used in recent studies. For instance, 10 out of 15 studies published from 2012 to 2019 utilized some type of haptic feedback (i.e., vibrotactile, electrotactile, electrocutaneous, or intraneural stimulation) when compared to two out of 16 studies during years 1975 to 2011.

Most studies assessed the performance of the BFB systems under laboratory conditions either walking on a treadmill or over ground. Most studies (above 50\%) also performed only one gait training session in which BFB was delivered to the participants. Most studies compared subject's gait performance with and without wearing the BFB system, walking at self-selected speed. Most of the studies did not report any follow-up sessions with the BFB system to test for retention. In addition, few studies evaluated changes on metabolic consumption [34,59], physical fatigue [59], and cognitive load or mental effort [58,59]. Most studies presented positive gait outcomes that were related to one or more physical and physiological parameters. However, six $(n=6)$ studies $[34,36,57,83-85]$ reported mixed results, showing gait improvements for some participants and not others after BFB. None of the studies reported negative effects BFB on gait. Only two studies $[84,85]$ reported non-persistent lasting effects and/or periods of retention after training. Table 5 details the key information that was retrieved from each article. 
Table 5. Key data extracted from the reviewed articles $(n=31)$.

\begin{tabular}{|c|c|c|c|c|c|c|c|c|}
\hline \multirow[b]{2}{*}{$\begin{array}{c}\text { Study } \\
\text { Characteristics }\end{array}$} & \multirow[b]{2}{*}{$\begin{array}{l}\text { Participant's } \\
\text { Characteristics }\end{array}$} & \multirow[b]{2}{*}{$\begin{array}{l}\text { Biofeedback (BFB) } \\
\text { Design }\end{array}$} & \multirow[b]{2}{*}{ Testing Conditions } & \multicolumn{3}{|c|}{ Outcome Measures } & \multirow[b]{2}{*}{$\begin{array}{c}\text { Intervention } \\
\text { Protocol Summary }\end{array}$} & \multirow[b]{2}{*}{ Key Findings } \\
\hline & & & & Gait Parameters & $\begin{array}{c}\text { Physical, } \\
\text { Physiological and } \\
\text { Other Parameters }\end{array}$ & Questionnaire & & \\
\hline $\begin{array}{l}\text { [59] } \\
\text { Petrini et al. } 2019 \\
\text { Real-time intraneural } \\
\text { stimulation to restore } \\
\text { sensory feedback of } \\
\text { transfemoral } \\
\text { amputees }\end{array}$ & $\begin{array}{l}2 \mathrm{TF} \\
\text { Cause: trauma } \\
\text { Age: } 49 \text { yrs, } 35 \text { yrs } \\
\text { PE: N/D } \\
\text { TSA: } 3 \text { yrs, } 12 \text { yrs }\end{array}$ & $\begin{array}{l}\text { FM: Intraneural } \\
\text { stimulation (touch, } \\
\text { pressure, or vibration) } \\
\text { FD: Surgical } \\
\text { implanted electrodes } \\
\text { FS: Concurrent } \\
\text { S/T: Insole pressure } \\
\text { sensors, knee encoder }\end{array}$ & $\begin{array}{l}\text { Lab \& Field, } \\
\text { Treadmill \& } \\
\text { Overground }\end{array}$ & $\begin{array}{l}\text { Heel-strike, flat foot, } \\
\text { toe-off, knee angle, } \\
\text { walking speed }\end{array}$ & $\begin{array}{l}\text { Metabolic } \\
\text { consumption }\left(\mathrm{VO}_{2}\right) \text {, } \\
\text { mental effort, } \\
\text { phantom limb pain }\end{array}$ & $\begin{array}{l}\text { Neuropathic Pain } \\
\text { Symptom Inventory } \\
\text { (NPSI), Visual Analog } \\
\text { Scale (VAS) }\end{array}$ & $\begin{array}{l}\text { Walking speed and } \\
\text { mental effort: } \\
6 \text { min outdoor (sand) } \\
\text { walking } \times 2 \text { sessions } \\
\text { per condition } \\
\text { (with/without } \\
\text { feedback + dual task), } \\
\text { Metabolic cost: } \\
\text { Indoors: (i) } 15 \text { min } \\
\text { treadmill walking } \\
\text { with increasing speed, } \\
\text { (ii) outdoors (grass): } 3 \\
\text { min baseline } x 6 \text { min } \\
\text { walking at SS speed }\end{array}$ & $\begin{array}{l}\text { Walking speed and } \\
\text { self-reported } \\
\text { confidence increased. } \\
\text { Mental and physical } \\
\text { fatigue decreased, } \\
\text { including reduced } \\
\text { phantom limb pain } \\
\text { with feedback }\end{array}$ \\
\hline $\begin{array}{l}\text { [57] } \\
\text { Fiedler et al. } 2019 \\
\text { Mobile visual } \\
\text { feedback system for } \\
\text { gait rehabilitation in } \\
\text { everyday-life } \\
\text { environment }\end{array}$ & $\begin{array}{l}1 \mathrm{TT} \\
\text { Cause: N/D } \\
\text { Age: } 61 \text { yrs } \\
\text { PE: } 12 \text { yrs } \\
\text { TSA: N/D }\end{array}$ & $\begin{array}{l}\text { FM: Visual } \\
\text { FD: Smart glasses } \\
\text { FS: Concurrent } \\
\text { S/T: Load cell }\end{array}$ & $\begin{array}{l}\text { Lab, } \\
\text { Overground }\end{array}$ & $\begin{array}{l}\text { Stance/step ratio, gait } \\
\text { symmetry index }\end{array}$ & N/A & $\mathrm{N} / \mathrm{A}$ & $\begin{array}{l}30 \mathrm{~m} \text { walking } \\
\text { (repeatedly) at SS } \\
\text { speed within 1-hr }\end{array}$ & $\begin{array}{l}\text { A strong correlation } \\
\text { found between } \\
\text { stance/step ratio (the } \\
\text { feedback variable) } \\
\text { and gait symmetry } \\
\text { index }\end{array}$ \\
\hline $\begin{array}{l}\text { [80] } \\
\text { Petrini et al. } 2019 \\
\text { Real-time tactile and } \\
\text { proprioceptive } \\
\text { feedback to increase } \\
\text { prosthesis } \\
\text { embodiment and to } \\
\text { improve mobility of } \\
\text { transfemoral } \\
\text { amputees }\end{array}$ & $\begin{array}{l}3 \mathrm{TF} \\
\text { Cause: trauma } \\
\text { Age: N/D } \\
\text { PE: N/D } \\
\text { TSA: } 3 \text { yrs, } 7 \text { yrs, } 12 \text { yrs }\end{array}$ & $\begin{array}{l}\text { FM: Intraneural } \\
\text { stimulation (touch, } \\
\text { pressure, or vibration) } \\
\text { FD: Surgical } \\
\text { implanted electrodes } \\
\text { FS: Concurrent } \\
\text { S/T: Insole pressure } \\
\text { sensors, knee encoder }\end{array}$ & $\begin{array}{l}\text { Lab, } \\
\text { Overground }\end{array}$ & $\begin{array}{l}\text { Heel-strike, flat foot, } \\
\text { toe-off, knee angle, } \\
\text { walking speed }\end{array}$ & $\begin{array}{l}\text { Error walking on a } \\
\text { line (walking agility), } \\
\text { proprioceptive } \\
\text { displacement, } \\
\text { cognitive load } \\
\text { (dual-task paradigm) }\end{array}$ & $\begin{array}{l}\text { Embodiment } \\
\text { questionnaire }\end{array}$ & $\begin{array}{l}\text { Nine } 5 \mathrm{~m} \text { walking } \\
\text { trials with/without } \\
\text { feedback over a } \\
\text { straight line (one foot } \\
\text { after the other } \\
\text { without stepping } \\
\text { outside the line) }\end{array}$ & $\begin{array}{l}\text { Improved mobility, } \\
\text { ease of cognitive } \\
\text { effort, and increased } \\
\text { embodiment of } \\
\text { prosthesis with } \\
\text { feedback }\end{array}$ \\
\hline $\begin{array}{l}\text { [35] } \\
\text { Brandt et al. } 2019 \\
\text { Visual feedback to } \\
\text { increase stance time } \\
\text { on the prosthetic limb. } \\
\text { Compare powered } \\
\text { versus passive knee } \\
\text { prostheses }\end{array}$ & $\begin{array}{l}5 \text { TF or knee disarticulation } \\
\text { Cause: } \\
\text { trauma/cancer/congenital } \\
\text { Age: } 19-59 \text { yrs } \\
\text { PE: } 6 \text { mos.-6 yrs } \\
\text { TSA: } 4-47 \text { yrs }\end{array}$ & $\begin{array}{l}\text { FM: Visual } \\
\text { FD: Computer } \\
\text { monitor } \\
\text { FS: Concurrent } \\
\text { S/T: Instrumented } \\
\text { treadmill (dual belt) } \\
\text { with force plates, } \\
\text { motion capture } \\
\text { system }\end{array}$ & $\begin{array}{l}\text { Lab, } \\
\text { Treadmill }\end{array}$ & $\begin{array}{l}\text { Stance time, swing } \\
\text { time, stance time } \\
\text { asymmetry, peak } \\
\text { anterior-posterior } \\
\text { ground reaction } \\
\text { forces, peak anterior } \\
\text { propulsive } \\
\text { asymmetry }\end{array}$ & $\mathrm{N} / \mathrm{A}$ & $\begin{array}{l}\text { Likert scale } \\
\text { (perceived difficulty) }\end{array}$ & $\begin{array}{l}\text { Twelve } 1.5 \mathrm{~min} \\
\text { walking trials at SS } \\
\text { speed with } 2 \text { min of } \\
\text { rest between trials } \\
\text { over } 3 \text { sessions of } 3 \mathrm{~h} \\
\text { each. Fitting and } \\
\text { training provided } \\
\text { during prior sessions. }\end{array}$ & $\begin{array}{l}\text { Stance time symmetry } \\
\text { and peak propulsion } \\
\text { symmetry } \\
\text { significantly } \\
\text { improved with both } \\
\text { prosthesis by } \\
\text { increasing prosthetic } \\
\text { stance time via } \\
\text { feedback }\end{array}$ \\
\hline
\end{tabular}


Table 5. Cont.

\begin{tabular}{|c|c|c|c|c|c|c|c|c|}
\hline \multirow[b]{2}{*}{$\begin{array}{c}\text { Study } \\
\text { Characteristics }\end{array}$} & \multirow[b]{2}{*}{$\begin{array}{l}\text { Participant's } \\
\text { Characteristics }\end{array}$} & \multirow[b]{2}{*}{$\begin{array}{c}\text { Biofeedback (BFB) } \\
\text { Design }\end{array}$} & \multirow[b]{2}{*}{ Testing Conditions } & \multicolumn{3}{|c|}{ Outcome Measures } & \multirow[b]{2}{*}{$\begin{array}{c}\text { Intervention } \\
\text { Protocol Summary }\end{array}$} & \multirow[b]{2}{*}{ Key Findings } \\
\hline & & & & Gait Parameters & $\begin{array}{c}\text { Physical, } \\
\text { Physiological and } \\
\text { Other Parameters }\end{array}$ & Questionnaire & & \\
\hline $\begin{array}{l}\text { [86] } \\
\text { Dietrich et al. } 2018 \\
\text { Assess whether } \\
\text { prostheses with } \\
\text { somatosensory } \\
\text { feedback can reduce } \\
\text { phantom limb pain } \\
\text { and increase } \\
\text { ambulation }\end{array}$ & $\begin{array}{l}14 \mathrm{TT} \\
\text { Cause: trauma/embolism } \\
\text { Age: } 27-76 \text { yrs } \\
\text { (56.3 } \pm 11.6 \mathrm{yrs}) \\
\text { PE: N/D } \\
\text { TSA: } 1-54 \text { yrs }\end{array}$ & $\begin{array}{l}\text { FM: Electrocutaneous } \\
\text { FD: Electrodes } \\
\text { FS: Concurrent } \\
\text { S/T: IIsole pressure } \\
\text { sensors }\end{array}$ & $\begin{array}{l}\text { Field, } \\
\text { Overground }\end{array}$ & Stance time & $\begin{array}{l}\text { Walking distance, } \\
\text { walking speed, } \\
\text { phantom limb pain }\end{array}$ & $\begin{array}{l}\text { Likert scale } \\
\text { (discrimination } \\
\text { performance), } \\
\text { Houghton Score } \\
\text { Questionnaire (HSQ), } \\
\text { Locomotor Capability } \\
\text { Index (LCI), Trinity } \\
\text { Amputation and } \\
\text { Experience Scales } \\
\text { (TAPES), Amputee } \\
\text { Body Image Scale } \\
\text { (ABIS), Pain } \\
\text { questionnaires, and } \\
\text { pain daily reports }\end{array}$ & $\begin{array}{l}10 \text { days of training } \\
\text { (walking at level } \\
\text { ground and uneven } \\
\text { terrains) over } 2 \\
\text { weeks, } 2 \text { sessions per } \\
\text { day, } 2 \mathrm{~h} \text { per session } \\
\text { with } 30-60 \text { min of rest } \\
\text { between daily } \\
\text { sessions. }\end{array}$ & $\begin{array}{l}\text { Reduction of } \\
\text { phantom limb pain, } \\
\text { larger walking } \\
\text { distances, stable } \\
\text { walking and better } \\
\text { posture control on } \\
\text { uneven ground with } \\
\text { feedback }\end{array}$ \\
\hline $\begin{array}{l}\text { [34] } \\
\text { Esposito et al. } 2017 \\
\text { Assess whether visual } \\
\text { feedback can reduce } \\
\text { center of mass sway } \\
\text { and metabolic } \\
\text { consumption during } \\
\text { gait retraining }\end{array}$ & $\begin{array}{l}\text { Study group: } \\
8 \mathrm{TT} \\
\text { Cause: trauma } \\
\text { Age: } 32.9 \pm 5.7 \mathrm{yrs} \\
\text { PE: } 29 \pm 38 \mathrm{mos} \\
\text { TSA: N/D } \\
\text { Control group: } \\
8 \mathrm{H} \\
\text { Cause: N/A } \\
\text { Age: } 29.4 \pm 3.8 \mathrm{yrs} \\
\text { PE: N/A } \\
\text { TSA: N/A }\end{array}$ & $\begin{array}{l}\text { FM: Visual (virtual } \\
\text { reality) } \\
\text { FD: CAREN } \\
\text { (Computer Assisted } \\
\text { Rehabilitation } \\
\text { Environment) } \\
\text { FS: Concurrent } \\
\text { S/T: Bipolar surface } \\
\text { electrodes, motion } \\
\text { capture system }\end{array}$ & $\begin{array}{l}\text { Lab, } \\
\text { Treadmill }\end{array}$ & Center of mass sway & $\begin{array}{l}\text { Metabolic rate }\left(\mathrm{VO}_{2}\right) \text {, } \\
\text { heart rate, thigh } \\
\text { muscle activation } \\
\text { magnitudes and } \\
\text { duration, quadriceps } \\
\text { and hamstrings } \\
\text { muscle activity }\end{array}$ & N/A & $\begin{array}{l}\text { Baseline: } 10 \mathrm{~min} \text { in } \\
\text { seated position ( } \mathrm{VO}_{2} \\
\text { baseline). } \\
\text { Acclimation: } 4 \text { min } \\
\text { practice receiving } \\
\text { visual feedback and } \\
\text { verbal cues (PT). } \\
\text { Data collection: } 8 \text { min } \\
\text { walking } \\
\text { (with/without visual } \\
\text { feedback) at } \\
\text { standardized speed }\end{array}$ & $\begin{array}{l}\text { Visual feedback } \\
\text { decreased center of } \\
\text { mass sway and } \\
\text { quadriceps activity. } \\
\text { Thigh muscle } \\
\text { co-contraction indices } \\
\text { unchanged. } \\
\text { Metabolic rate was } \\
\text { not significantly } \\
\text { affected by feedback }\end{array}$ \\
\hline $\begin{array}{l}\text { [87] } \\
\text { Maldonado et al. } \\
2017 \\
\text { BFB system } \\
\text { developed as a } \\
\text { training tool to sense } \\
\text { perturbations to } \\
\text { perform corrective } \\
\text { actions to avoid falls }\end{array}$ & $\begin{array}{l}2 \text { TT } \\
\text { Cause: N/D } \\
\text { Age: } 49 \text { yrs, } 67 \text { yrs } \\
\text { PE: N/D } \\
\text { TSA: N/D }\end{array}$ & $\begin{array}{l}\text { FM: Vibrotactile } \\
\text { FD: Vibrating motors, } \\
\text { solenoid } \\
\text { FS: Concurrent } \\
\text { S/T: } \\
\text { Electrogoniometer }\end{array}$ & $\begin{array}{l}\text { Lab, } \\
\text { Overground }\end{array}$ & Knee angle & $\begin{array}{l}\text { Reaction times, } \\
\text { subject's response to } \\
\text { stimulus }\end{array}$ & $\mathrm{N} / \mathrm{A}$ & $\begin{array}{l}\text { Six } 1 \mathrm{~h} \text { to } 2 \mathrm{~h} \text { training } \\
\text { sessions over } 3 \text { weeks, } \\
\text { receiving only } \\
\text { vibrotactile feedback. } \\
\text { One } 2 \mathrm{~h} \text { session, } \\
\text { vibrotactile and } \\
\text { solenoid feedback } \\
\text { (retention and } \\
\text { transfer test) }\end{array}$ & $\begin{array}{l}\text { Subjects performed } \\
\text { the corrective } \\
\text { movement in } \\
\text { response to feedback. } \\
\text { No conclusive results } \\
\text { for retention and } \\
\text { transfer tests. }\end{array}$ \\
\hline
\end{tabular}


Table 5. Cont

\begin{tabular}{|c|c|c|c|c|c|c|c|c|}
\hline \multirow[b]{2}{*}{$\begin{array}{c}\text { Study } \\
\text { Characteristics }\end{array}$} & \multirow[b]{2}{*}{$\begin{array}{c}\text { Participant's } \\
\text { Characteristics }\end{array}$} & \multirow[b]{2}{*}{$\begin{array}{l}\text { Biofeedback (BFB) } \\
\text { Design }\end{array}$} & \multirow[b]{2}{*}{ Testing Conditions } & \multicolumn{3}{|c|}{ Outcome Measures } & \multirow[b]{2}{*}{$\begin{array}{c}\text { Intervention } \\
\text { Protocol Summary }\end{array}$} & \multirow[b]{2}{*}{ Key Findings } \\
\hline & & & & Gait Parameters & $\begin{array}{c}\text { Physical, } \\
\text { Physiological and } \\
\text { Other Parameters }\end{array}$ & Questionnaire & & \\
\hline $\begin{array}{l}\text { [58] } \\
\text { Crea et al. } 2017 \\
\text { BFB system } \\
\text { developed to improve } \\
\text { temporal gait } \\
\text { symmetry of elderly } \\
\text { transfemoral } \\
\text { amputees }\end{array}$ & $\begin{array}{l}3 \mathrm{TF} \\
\text { Cause: N/D } \\
\text { Age: }>60 \mathrm{yrs} \\
\text { PE: N/D } \\
\text { TSA: }>1 \text { year }\end{array}$ & $\begin{array}{l}\text { FM: Vibrotactile, } \\
\text { Visual } \\
\text { FD: Vibrating motors, } \\
\text { display screen } \\
\text { FS: Concurrent } \\
\text { S/T: } \\
\text { Pressure-sensitive } \\
\text { insoles }\end{array}$ & $\begin{array}{l}\text { Lab, } \\
\text { Treadmill }\end{array}$ & $\begin{array}{l}\text { Stance time, } \\
\text { symmetry index, } \\
\text { cadence }\end{array}$ & $\begin{array}{l}\text { Heart rate, breathing } \\
\text { rate, skin } \\
\text { temperature, skin } \\
\text { conductance, } \\
\text { cognitive load }\end{array}$ & $\begin{array}{l}\text { National Aeronautics } \\
\text { and Space } \\
\text { Administration Task } \\
\text { Load Index } \\
\text { (NASA-TLX34), } \\
\text { System Usability } \\
\text { Scale (SUS) }\end{array}$ & $\begin{array}{l}\text { Within a week: Pre- } \\
\text { and Post-training, } 1 \\
\text { session each (only } \\
\text { vibrotactile). } 3 \\
\text { sessions training } \\
\text { (vibrotactile + visual } \\
\text { feedback). Follow-up } \\
\text { a week after (only } \\
\text { vibrotactile) }\end{array}$ & $\begin{array}{l}\text { Feedback improved } \\
\text { symmetry index and } \\
\text { lower cadence } \\
\text { promoting longer } \\
\text { strides. Cognitive } \\
\text { load did not increase } \\
\text { with feedback. } \\
\text { No signs of negative } \\
\text { psychophysiological } \\
\text { effects. }\end{array}$ \\
\hline $\begin{array}{l}{[28]} \\
\text { Plauche et al. } 2016 \\
\text { Develop a BFB } \\
\text { system to asses gait } \\
\text { performance under } \\
\text { different vibrotactile } \\
\text { feedbacks strategies on } \\
\text { able-bodied subjects } \\
\text { walking with a } \\
\text { prosthetic adaptor }\end{array}$ & $\begin{array}{l}9 \mathrm{H} \text { (above-knee prosthetic } \\
\text { adaptor) } \\
\text { Cause: N/A } \\
\text { Age: } 25.6 \pm 2 \text { yrs } \\
\text { PE: N/A } \\
\text { TSA: N/A }\end{array}$ & $\begin{array}{l}\text { FM: Vibrotactile } \\
\text { FD: Vibrating motors } \\
\text { FS: Concurrent } \\
\text { S/T: Force sensing } \\
\text { resistors (FSRs) } \\
\text { sensors }\end{array}$ & $\begin{array}{l}\text { Lab, } \\
\text { Treadmill }\end{array}$ & $\begin{array}{l}\text { Stride length step } \\
\text { width, trunk sway, } \\
\text { including their } \\
\text { variabilities }\end{array}$ & $\mathrm{N} / \mathrm{A}$ & $\begin{array}{l}\text { Likert scale (feedback } \\
\text { strategies) }\end{array}$ & $\begin{array}{l}\text { Walking } 30 \text { s at SS } \\
\text { speed on a treadmill } \\
\text { (10 trials per } \\
\text { condition) } \\
\text { with/without } \\
\text { feedback and } \\
\text { with/without } \\
\text { prosthesis adaptor }\end{array}$ & $\begin{array}{l}\text { Improved gait } \\
\text { stability as the } \\
\text { variability of stride } \\
\text { length, step width } \\
\text { and trunk sway } \\
\text { decreased. }\end{array}$ \\
\hline $\begin{array}{l}\text { [84] } \\
\text { Pagel et al. 2016 } \\
\text { Develop a BFB } \\
\text { system to improve } \\
\text { gait symmetry by } \\
\text { providing feedback } \\
\text { from foot center of } \\
\text { pressure and knee } \\
\text { flexion angle }\end{array}$ & $\begin{array}{l}3 \mathrm{TF} \\
\text { Cause: trauma/cancer } \\
\text { Age: } 21 \text { yrs, } 54 \text { yrs, } 73 \mathrm{yrs} \\
\text { PE: } 1 \text { yrs, } 36 \text { yrs, } 53 \text { yrs } \\
\text { TSA: } 1 \text { yrs, } 52 \text { yrs, } 53 \text { yrs }\end{array}$ & $\begin{array}{l}\text { FM: Electrotactile } \\
\text { FD: Electrodes } \\
\text { FS: Concurrent } \\
\text { S/T: Force/moment } \\
\text { sensor, } \\
\text { goniometer-gyroscope } \\
\text { sensor }\end{array}$ & $\begin{array}{l}\text { Lab, } \\
\text { Treadmill }\end{array}$ & $\begin{array}{l}\text { Stance time, step } \\
\text { length, stance time } \\
\text { ratio, step length } \\
\text { ratio, ground reaction } \\
\text { forces, center of } \\
\text { pressure (CoP), knee } \\
\text { flexion angle }\end{array}$ & $\mathrm{N} / \mathrm{A}$ & $\begin{array}{l}\text { User's feedback } \\
\text { experience } \\
\text { questionnaire }\end{array}$ & $\begin{array}{l}2 \text { min walking per } \\
\text { condition (no } \\
\text { feedback, CoP } \\
\text { feedback, and knee } \\
\text { angle feedback), SS } \\
\text { speed }\end{array}$ & $\begin{array}{l}\text { No persistent positive } \\
\text { effect but improved } \\
\text { step length for one } \\
\text { participant. } \\
\text { Subjects felt more } \\
\text { benefited from knee } \\
\text { angle feedback than } \\
\text { CoP feedback. }\end{array}$ \\
\hline $\begin{array}{l}\text { [82] } \\
\text { Huang et al. } 2016 \\
\text { Utilize visual } \\
\text { feedback to alter } \\
\text { prosthetic ankle } \\
\text { performance while } \\
\text { using a powered } \\
\text { prosthesis with } \\
\text { myoelectric } \\
\text { controlled }\end{array}$ & $\begin{array}{l}5 \mathrm{TT} \\
\text { Cause: trauma/cancer } \\
\text { Age: } 23-70 \text { yrs }(55.4 \pm 18.6 \\
\text { yrs) } \\
\text { PE: N/D } \\
\text { TSA: } 4-44 \text { yrs }(22.6 \pm 19 \\
\text { yrs) }\end{array}$ & $\begin{array}{l}\text { FM: Visual } \\
\text { FD: Computer } \\
\text { monitor } \\
\text { FS: Concurrent } \\
\text { S/T: Motion capture } \\
\text { system, force plates, } \\
\text { electromyography } \\
\text { (EMG) sensors }\end{array}$ & $\begin{array}{l}\text { Lab, } \\
\text { Treadmill }\end{array}$ & $\begin{array}{l}\text { Peak ankle power, } \\
\text { total ankle work, } \\
\text { positive ankle work, } \\
\text { negative ankle work }\end{array}$ & $\begin{array}{l}\text { Residual limb muscle } \\
\text { activation patterns }\end{array}$ & N/A & $\begin{array}{l}5 \text { min to } 10 \mathrm{~min} \\
\text { walking trial with } \\
\text { prescribed and } \\
\text { powered prosthesis } \\
\text { with/without } \\
\text { feedback, speed } 1.0 \\
\mathrm{~m} / \mathrm{s} . \\
\text { An average of } 3.5 \mathrm{~h} \text { of } \\
\text { training in total over } \\
2 \text { months. } \\
\end{array}$ & $\begin{array}{l}\text { Adapted muscle } \\
\text { activation patterns } \\
\text { due to visual } \\
\text { feedback. Increased } \\
\text { peak ankle power and } \\
\text { positive ankle work. }\end{array}$ \\
\hline
\end{tabular}


Table 5. Cont

\begin{tabular}{|c|c|c|c|c|c|c|c|c|}
\hline \multirow[b]{2}{*}{$\begin{array}{c}\text { Study } \\
\text { Characteristics }\end{array}$} & \multirow[b]{2}{*}{$\begin{array}{l}\text { Participant's } \\
\text { Characteristics }\end{array}$} & \multirow[b]{2}{*}{$\begin{array}{l}\text { Biofeedback (BFB) } \\
\text { Design }\end{array}$} & \multirow[b]{2}{*}{ Testing Conditions } & \multicolumn{3}{|c|}{ Outcome Measures } & \multirow[b]{2}{*}{$\begin{array}{c}\text { Intervention } \\
\text { Protocol Summary }\end{array}$} & \multirow[b]{2}{*}{ Key Findings } \\
\hline & & & & Gait Parameters & $\begin{array}{c}\text { Physical, } \\
\text { Physiological and } \\
\text { Other Parameters }\end{array}$ & Questionnaire & & \\
\hline $\begin{array}{l}\text { [30] } \\
\text { Crea et al. } 2015 \\
\text { BFB system to } \\
\text { provide vibrotactile } \\
\text { feedback during } \\
\text { gait-phase transitions }\end{array}$ & $\begin{array}{l}10 \mathrm{H} \\
\text { Cause: N/A } \\
\text { Age: } 27 \pm 1.8 \text { yrs } \\
\text { PE: N/A } \\
\text { TSA: N/A }\end{array}$ & $\begin{array}{l}\text { FM: Vibrotactile } \\
\text { FD: Vibrating motors } \\
\text { FS: Concurrent } \\
\text { S/T: } \\
\text { Pressure-sensitive } \\
\text { insoles }\end{array}$ & $\begin{array}{l}\text { Lab, } \\
\text { Treadmill }\end{array}$ & $\begin{array}{l}\text { Stance time, swing } \\
\text { time, step cadence, } \\
\text { vertical ground } \\
\text { reaction force, center } \\
\text { of pressure }\end{array}$ & N/A & $\begin{array}{l}\text { Self-assessment } \\
\text { questionnaire } \\
\text { (cognitive effort) }\end{array}$ & $\begin{array}{l}6 \text { min walking per } \\
\text { condition (missing } \\
\text { stimuli, delay stimuli: } \\
200 \mathrm{~s} \& 500 \mathrm{~s} \text {, and } \\
\text { wrong stimuli). }\end{array}$ & $\begin{array}{l}\text { Accuracy in stimuli } \\
\text { detection decreased if } \\
\text { delay increased. } \\
\text { Good usability, } \\
\text { feedback is readily } \\
\text { perceived by } \\
\text { participants. }\end{array}$ \\
\hline $\begin{array}{l}{[77]} \\
\text { Lee et al. 2013 } \\
\text { Evaluate a BFB } \\
\text { system using } \\
\text { subsensory } \\
\text { stimulation and } \\
\text { visual-auditory } \\
\text { feedback to improve } \\
\text { postural sway and } \\
\text { dynamic weight } \\
\text { shifting stability }\end{array}$ & $\begin{array}{l}7 \mathrm{TT} \\
\text { Cause: N/D } \\
\text { Age: } 24-60 \text { yrs }(38.8 \pm \\
14.08 \text { yrs) } \\
\text { PE: > } 2 \text { yrs }(8.5 \pm 6.12 \text { yrs }) \\
\text { TSA: N/D }\end{array}$ & $\begin{array}{l}\text { FM: Auditory, Visual } \\
\text { FD: PC speaker, } \\
\text { computer monitor } \\
\text { FS: Concurrent } \\
\text { S/T: Force sensing } \\
\text { resistors (FSRs) } \\
\text { sensors }\end{array}$ & $\begin{array}{l}\text { Lab, } \\
\text { Treadmill }\end{array}$ & $\begin{array}{l}\text { Double support time } \\
\text { symmetry index, } \\
\text { constant time step } \\
\text { number index, single } \\
\text { support time } \\
\text { symmetry index, gait } \\
\text { phase time ratio index }\end{array}$ & Heart rate & $\mathrm{N} / \mathrm{A}$ & $\begin{array}{l}20 \text { min each test } \\
\text { session (5 min warm } \\
\text { up, } 10 \text { min training } \\
\text { and } 5 \text { min cool down). } \\
\text { Walking speed } \\
\text { increased each } \\
\text { minute as tolerated } \\
\text { (starting at SS speed) }\end{array}$ & $\begin{array}{l}\text { Improvement in } \\
\text { weight shifting } \\
\text { stability indices. } \\
\text { Most subjects easily } \\
\text { adapted to auditory } \\
\text { rather than visual } \\
\text { biofeedback. }\end{array}$ \\
\hline $\begin{array}{l}\text { [88] } \\
\text { Redd et al. } 2012 \\
\text { Assess the ability of a } \\
\text { BFB system to alter } \\
\text { gait symmetry under } \\
\text { visual, auditory and } \\
\text { vibrotactile feedback }\end{array}$ & $\begin{array}{l}12 \mathrm{H} \\
\text { Cause: N/A } \\
\text { Age: N/D } \\
\text { PE: N/A } \\
\text { TSA: N/A }\end{array}$ & $\begin{array}{l}\text { FM: Auditory, } \\
\text { Vibrotactile, Visual } \\
\text { FD: Smartphone } \\
\text { FS: Concurrent } \\
\text { S/T: Force sensing } \\
\text { resistors (FSRs) } \\
\text { sensors }\end{array}$ & $\begin{array}{l}\text { Lab, } \\
\text { Overground }\end{array}$ & $\begin{array}{l}\text { Stance time symmetry } \\
\text { ratio }\end{array}$ & N/A & Usability survey & $\begin{array}{l}\text { Six } 200 \mathrm{ft} \text { walking } \\
\text { trials (one trial per } \\
\text { feedback modality } \\
\text { and } 3 \text { trials with the } \\
\text { preferred feedback } \\
\text { modality) }\end{array}$ & $\begin{array}{l}\text { BFB altered gait of } \\
\text { user without } \\
\text { supervision from a } \\
\text { specialist. } \\
\text { Visual was the } \\
\text { preferred feedback } \\
\text { modality. }\end{array}$ \\
\hline $\begin{array}{l}\text { [36] } \\
\text { Yang et al. 2012 } \\
\text { Evaluate the } \\
\text { performance of a BFB } \\
\text { device to improve } \\
\text { gait symmetry of } \\
\text { prosthetic users }\end{array}$ & $\begin{array}{l}3 \text { TT } \\
\text { Cause: infection/embolism } \\
\text { Age: } 22-65 \text { yrs }(49.7 \pm 19.6 \\
\text { yrs) } \\
\text { PE: N/D } \\
\text { TSA: } 7 \text { mos. }-5.5 \text { yrs }\end{array}$ & $\begin{array}{l}\text { FM: Auditory } \\
\text { FD: BFB buzzer } \\
\text { FS: Concurrent } \\
\text { S/T: Force sensing } \\
\text { resistors (FSRs) } \\
\text { sensors, motion } \\
\text { capture system, force } \\
\text { plates }\end{array}$ & $\begin{array}{l}\text { Lab, } \\
\text { N/D }\end{array}$ & $\begin{array}{l}\text { Stance time, } \\
\text { symmetry ratio, trunk } \\
\text { sway }\end{array}$ & N/A & N/A & $\begin{array}{l}\text { Pre-test } 1 \text { week before, } \\
\text { six } 30 \text { min training, } \\
\text { post-test } 1 \text { week after. } \\
\text { PT set trial duration } \\
\text { (avg. 30s-240s) and } \\
\text { feedback thresholds. }\end{array}$ & $\begin{array}{l}2 \text { of } 3 \text { subjects } \\
\text { improved symmetry } \\
\text { ratio and trunk sway }\end{array}$ \\
\hline $\begin{array}{l}\text { [81] } \\
\text { Darter et al. } 2011 \\
\text { Assess biomechanical } \\
\text { and physiological } \\
\text { effects of gait training } \\
\text { using virtual reality }\end{array}$ & $\begin{array}{l}1 \mathrm{TF} \\
\text { Cause: trauma } \\
\text { Age: } 24 \text { yrs } \\
\text { PE: } 2 \text { yrs } \\
\text { TSA: N/D }\end{array}$ & $\begin{array}{l}\text { FM: Visual (virtual } \\
\text { reality) } \\
\text { FD: CAREN } \\
\text { (Computer Assisted } \\
\text { Rehabilitation } \\
\text { Environment) } \\
\text { FS: Concurrent, } \\
\text { verbal cues (PT) } \\
\text { S/T: Motion capture } \\
\text { system, force plates }\end{array}$ & $\begin{array}{l}\text { Lab, Treadmill \& } \\
\text { Overground }\end{array}$ & $\begin{array}{l}\text { Frontal-plane trunk } \\
\text { motion, frontal plane } \\
\text { hip, pelvis and trunk } \\
\text { angles, walking } \\
\text { speed, step length, } \\
\text { stance time, step } \\
\text { width }\end{array}$ & $\mathrm{VO}_{2}$ consumption & N/A & $\begin{array}{l}\text { Twelve } 30 \text { min } \\
\text { walking sessions } \\
\text { within } 3 \text { weeks. } \\
\text { Follow-up: } 3 \text { weeks } \\
\text { after training. } \\
\text { PT involved during } \\
\text { first BFB sessions }\end{array}$ & $\begin{array}{l}\text { Training effective in } \\
\text { improving frontal } \\
\text { plane hip, pelvis and } \\
\text { trunk motion, with } \\
\text { decreases in oxygen } \\
\text { consumption during } \\
\text { overground walking. } \\
\text { Retention found at } 3 \\
\text { weeks after training }\end{array}$ \\
\hline
\end{tabular}


Table 5. Cont

\begin{tabular}{|c|c|c|c|c|c|c|c|c|}
\hline \multirow[b]{2}{*}{$\begin{array}{c}\text { Study } \\
\text { Characteristics }\end{array}$} & \multirow[b]{2}{*}{$\begin{array}{c}\text { Participant's } \\
\text { Characteristics }\end{array}$} & \multirow[b]{2}{*}{$\begin{array}{l}\text { Biofeedback (BFB) } \\
\text { Design }\end{array}$} & \multirow[b]{2}{*}{ Testing Conditions } & \multicolumn{3}{|c|}{ Outcome Measures } & \multirow[b]{2}{*}{$\begin{array}{c}\text { Intervention } \\
\text { Protocol Summary }\end{array}$} & \multirow[b]{2}{*}{ Key Findings } \\
\hline & & & & Gait Parameters & $\begin{array}{c}\text { Physical, } \\
\text { Physiological and } \\
\text { Other Parameters }\end{array}$ & Questionnaire & & \\
\hline $\begin{array}{l}\text { [76] } \\
\text { Lee et al. 2010 } \\
\text { Asses a BFB system } \\
\text { using subsensory } \\
\text { stimulation and } \\
\text { visual-auditory } \\
\text { feedback to improve } \\
\text { postural sway and } \\
\text { dynamic weight } \\
\text { shifting stability }\end{array}$ & $\begin{array}{l}7 \text { TT } \\
\text { Cause: } \mathrm{N} / \mathrm{D} \\
\text { Age: } 24-60 \text { yrs }(38.8 \pm \\
14.08 \text { yrs) } \\
\text { PE }>2 \text { yrs }(8.5 \pm 6.12 \text { yrs) } \\
\text { TSA: N/D }\end{array}$ & $\begin{array}{l}\text { FM: Auditory, Visual } \\
\text { FD: PC speaker, } \\
\text { computer monitor } \\
\text { FS: Concurrent } \\
\text { S/T: Force sensing } \\
\text { resistors (FSRs) } \\
\text { sensors }\end{array}$ & $\begin{array}{l}\text { Lab, } \\
\text { Treadmill }\end{array}$ & $\begin{array}{l}\text { Double support time } \\
\text { symmetry index, } \\
\text { constant time step } \\
\text { number index, single } \\
\text { support time } \\
\text { symmetry index, gait } \\
\text { phase time ratio index }\end{array}$ & Heart rate & N/A & $\begin{array}{l}20 \mathrm{~min} \text { each test } \\
\text { session ( } 5 \mathrm{~min} \text { warm } \\
\text { up, } 10 \mathrm{~min} \text { training } \\
\text { and } 5 \mathrm{~min} \text { cool down). } \\
\text { Walking speed } \\
\text { increased each } \\
\text { minute as tolerated } \\
\text { (starting at SS speed) }\end{array}$ & $\begin{array}{l}\text { Improvement in } \\
\text { weight shifting } \\
\text { stability indices. } \\
\text { Most subjects easily } \\
\text { adapted to auditory } \\
\text { rather than visual } \\
\text { feedback }\end{array}$ \\
\hline $\begin{array}{l}\text { [75] } \\
\text { Lee et al. 2009 } \\
\text { Assess a BFB system } \\
\text { using subsensory } \\
\text { stimulation and } \\
\text { visual-auditory } \\
\text { feedback to improve } \\
\text { postural sway and } \\
\text { dynamic weight } \\
\text { shifting stability }\end{array}$ & $\begin{array}{l}7 \mathrm{TT} \\
\text { Cause: } \mathrm{N} / \mathrm{D} \\
\text { Age: } 24-60 \mathrm{yrs} \\
(38.8 \pm 14.08 \mathrm{yrs}) \\
\text { PE: }>2 \text { yrs }(8.5 \pm 6.12 \mathrm{yrs}) \\
\text { TSA: N/D }\end{array}$ & $\begin{array}{l}\text { FM: Auditory, Visual } \\
\text { FD: PC speaker, } \\
\text { computer monitor } \\
\text { FS: Concurrent } \\
\text { S/T: Force sensing } \\
\text { resistors (FSRs) } \\
\text { sensors }\end{array}$ & $\begin{array}{l}\text { Lab, } \\
\text { Treadmill }\end{array}$ & $\begin{array}{l}\text { Double support time } \\
\text { symmetry index, } \\
\text { constant time step } \\
\text { number index, single } \\
\text { support time } \\
\text { symmetry index, gait } \\
\text { phase time ratio index }\end{array}$ & Heart rate & $\mathrm{N} / \mathrm{A}$ & $\begin{array}{l}20 \text { min each test } \\
\text { session ( } 5 \text { min warm } \\
\text { up, } 10 \text { min training } \\
\text { and } 5 \text { min cool } \\
\text { down).Walking speed } \\
\text { increased each } \\
\text { minute as tolerated } \\
\text { (starting at SS speed) }\end{array}$ & $\begin{array}{l}\text { Improvement in } \\
\text { weight shifting } \\
\text { stability indices. } \\
\text { Most subjects easily } \\
\text { adapted to auditory } \\
\text { rather than visual } \\
\text { feedback }\end{array}$ \\
\hline $\begin{array}{l}{[74]} \\
\text { Lee et al. } 2008 \\
\text { Evaluate a } \\
\text { computerized foot } \\
\text { pressure BFB system } \\
\text { using subsensory } \\
\text { electrical stimulation } \\
\text { and visual-auditory } \\
\text { feedback to improve } \\
\text { gait and balance of } \\
\text { transtibial amputees }\end{array}$ & $\begin{array}{l}5 \mathrm{TT} \\
\text { Cause: N/D } \\
\text { Age: } 24-48 \text { yrs }(37.4 \pm 11.57 \\
\text { yrs) } \\
\text { PE: } 2 \text { yrs } \\
\text { TSA: N/D }\end{array}$ & $\begin{array}{l}\text { FM: Auditory, Visual } \\
\text { FD: PC speaker, } \\
\text { computer monitor } \\
\text { FS: Concurrent } \\
\text { S/T: Force sensing } \\
\text { resistors (FSRs) } \\
\text { sensors }\end{array}$ & $\begin{array}{l}\text { Lab, } \\
\text { Treadmill }\end{array}$ & $\begin{array}{l}\text { Double support time } \\
\text { index, constant time } \\
\text { cadence index, single } \\
\text { support time index, } \\
\text { stance/swing phase } \\
\text { index }\end{array}$ & Heart rate & N/A & $\begin{array}{l}20 \text { min each test } \\
\text { session }(5 \text { min warm } \\
\text { up, } 10 \text { min training } \\
\text { and } 5 \text { min cool down). } \\
\text { Walking speed } \\
\text { increased each } \\
\text { minute as tolerated } \\
\text { (starting at SS speed) }\end{array}$ & $\begin{array}{l}\text { Improvement in all } \\
\text { dynamic gait } \\
\text { performance indices } \\
\text { Most subjects easily } \\
\text { adapted to auditory } \\
\text { rather than visual } \\
\text { feedback }\end{array}$ \\
\hline $\begin{array}{l}\text { [56] } \\
\text { Lee et al. } 2007 \\
\text { Assess a } \\
\text { computerized foot } \\
\text { pressure BFB system } \\
\text { using low-level } \\
\text { electrical stimulation } \\
\text { and visual-auditory } \\
\text { feedback to improve } \\
\text { gait and balance }\end{array}$ & $\begin{array}{l}7 \text { TT } \\
\text { Cause: N/D } \\
\text { Age: } 24-60 \text { yrs }(38.8 \pm \\
14.08 \text { yrs) } \\
\text { PE: }>2 \text { yrs }(8.5 \pm 6.12 \text { yrs }) \\
\text { TSA: N/D }\end{array}$ & $\begin{array}{l}\text { FM: Auditory, Visual } \\
\text { FD: PC speaker, } \\
\text { computer monitor } \\
\text { FS: Concurrent } \\
\text { S/T: Force sensing } \\
\text { resistors (FSRs) } \\
\text { sensors }\end{array}$ & $\begin{array}{l}\text { Lab, } \\
\text { Treadmill }\end{array}$ & $\begin{array}{l}\text { Double support } \\
\text { period, constant time } \\
\text { cadence, single } \\
\text { support period, } \\
\text { stance/swing ratio }\end{array}$ & N/A & N/A & $\begin{array}{l}20 \text { min each test } \\
\text { session ( } 5 \text { min warm } \\
\text { up, } 10 \text { min training } \\
\text { and } 5 \text { min cool down). } \\
\text { Walking speed } \\
\text { increased each } \\
\text { minute as tolerated } \\
\text { (starting at SS speed) }\end{array}$ & $\begin{array}{l}\text { Improvement in all } \\
\text { dynamic gait } \\
\text { performance } \\
\text { measures. } \\
\text { Most subjects easily } \\
\text { adapted to auditory } \\
\text { rather than visual } \\
\text { feedback }\end{array}$ \\
\hline
\end{tabular}


Table 5. Cont.

\begin{tabular}{|c|c|c|c|c|c|c|c|c|}
\hline \multirow[b]{2}{*}{$\begin{array}{c}\text { Study } \\
\text { Characteristics }\end{array}$} & \multirow[b]{2}{*}{$\begin{array}{c}\text { Participant's } \\
\text { Characteristics }\end{array}$} & \multirow[b]{2}{*}{$\begin{array}{l}\text { Biofeedback (BFB) } \\
\text { Design }\end{array}$} & \multirow[b]{2}{*}{ Testing Conditions } & \multicolumn{3}{|c|}{ Outcome Measures } & \multirow[b]{2}{*}{$\begin{array}{c}\text { Intervention } \\
\text { Protocol Summary }\end{array}$} & \multirow[b]{2}{*}{ Key Findings } \\
\hline & & & & Gait Parameters & $\begin{array}{c}\text { Physical, } \\
\text { Physiological and } \\
\text { Other Parameters }\end{array}$ & Questionnaire & & \\
\hline $\begin{array}{l}\text { [66] } \\
\text { Isakov et al. } 2007 \\
\text { Evaluate the } \\
\text { effectiveness of a BFB } \\
\text { system compare to } \\
\text { traditional training } \\
\text { (control group) to } \\
\text { improve full } \\
\text { weight-bearing of } \\
\text { lower-limb amputees }\end{array}$ & $\begin{array}{l}42 \text { LLA } \\
\text { (TF, TT, hip and knee } \\
\text { replacement, femoral neck } \\
\text { fracture), } \\
\text { ( } \mathrm{n}=22 \text { study, } \mathrm{n}=20 \text { control } \\
\text { group) } \\
\text { Cause: N/D } \\
\text { Age: avg. } 62 \text { yrs (study), } 66 \\
\text { yrs (control) } \\
\text { PE: } \text { N/D } \\
\text { TSA: N/D }\end{array}$ & $\begin{array}{l}\text { FM: Auditory (study), } \\
\text { Verbal cues (control) } \\
\text { FD: SmartSte }{ }^{\mathrm{TM}} \\
\text { (audio) } \\
\text { FS: Concurrent } \\
\text { (study), } \\
\text { Physiotherapy } \\
\text { (control) } \\
\text { S/T: Pressure sensors } \\
\text { (study) }\end{array}$ & $\begin{array}{l}\mathrm{N} / \mathrm{D}, \\
\mathrm{N} / \mathrm{D}\end{array}$ & $\begin{array}{l}\text { Prosthetic } \\
\text { weight-bearing }\end{array}$ & N/A & $\mathrm{N} / \mathrm{A}$ & $\begin{array}{l}\text { Both groups: } 10 \mathrm{~m} \\
\text { walking at SS speed. } \\
\text { Four } 30 \text { min sessions } \\
\text { within } 14 \text { days. }\end{array}$ & $\begin{array}{l}\text { Weight-bearing on } \\
\text { the prosthetic limb } \\
\text { was statistically } \\
\text { significant increased } \\
\text { while using BFB }\end{array}$ \\
\hline $\begin{array}{l}\text { [89] } \\
\text { Davis et al. 2004 } \\
\text { Evaluate whether a } \\
\text { BFB system is capable } \\
\text { to reduce oxygen } \\
\text { consumption by } \\
\text { improving gait } \\
\text { symmetry of } \\
\text { lower-limb amputees }\end{array}$ & $\begin{array}{l}11 \mathrm{TF} / \mathrm{TT} \\
\text { Cause: trauma/diabetes } \\
\text { Age: } 36-58 \text { yrs } \\
\text { PE: N/D } \\
\text { TSA: N/D }\end{array}$ & $\begin{array}{l}\text { FM: Visual } \\
\text { FD: Computer } \\
\text { monitor } \\
\text { FS: Concurrent } \\
\text { S/T: Instrumented } \\
\text { treadmill with force } \\
\text { plates }\end{array}$ & $\begin{array}{l}\text { Lab, } \\
\text { Treadmill }\end{array}$ & $\begin{array}{l}\text { Stance/swing ratios, } \\
\text { foot propulsive forces, } \\
\text { shear foot forces }\end{array}$ & $\begin{array}{l}\text { Heart rate, } \mathrm{VO}_{2} \\
\text { consumption, tidal } \\
\text { volume }\end{array}$ & N/A & $\begin{array}{l}\text { Five } 4 \text { min tests } \\
\text { with/without } \\
\text { feedback per each } \\
\text { target gait parameter } \\
\text { (stance/swing ratio, } \\
\text { foot propulsive forces, } \\
\text { and shear foot forces) }\end{array}$ & $\begin{array}{l}\text { Real-time visual } \\
\text { feedback results in } \\
\text { immediate symmetry } \\
\text { improvements. } \\
\text { Significant reductions } \\
\text { in heart rate and } \\
\text { oxygen consumption } \\
\text { with feedback }\end{array}$ \\
\hline $\begin{array}{l}\text { [90] } \\
\text { Chow et al. 2000 } \\
\text { Evaluate the effects of } \\
\text { BFB on } \\
\text { weight-bearing } \\
\text { patterns of TT } \\
\text { amputees at early } \\
\text { postoperative period }\end{array}$ & $\begin{array}{l}6 \mathrm{TT} \\
\text { Cause: diabetes/peripheral } \\
\text { vascular disease } \\
\text { Age: } 66-78 \text { yrs } \\
\text { PE: N/D } \\
\text { TSA: N/D }\end{array}$ & $\begin{array}{l}\text { FM: Auditory } \\
\text { FD: BFB buzzer } \\
\text { FS: Concurrent } \\
\text { S/T: Load-monitoring } \\
\text { device (pair of } \\
\text { single-axis strain } \\
\text { gauges) }\end{array}$ & $\begin{array}{l}\text { Lab, } \\
\text { Overground }\end{array}$ & $\begin{array}{l}\text { Prosthetic } \\
\text { weight-bearing }\end{array}$ & $\mathrm{N} / \mathrm{A}$ & N/A & $\begin{array}{l}4 \text { randomized } \\
\text { walking trials (5m } \\
\text { length) with/without } \\
\text { feedback over } 5 \\
\text { sessions at SS speed }\end{array}$ & $\begin{array}{l}\text { Feedback prevents } \\
\text { overloading of the } \\
\text { residual limb beyond } \\
\text { the pre-set load target }\end{array}$ \\
\hline $\begin{array}{l}\text { [91] } \\
\text { Dingwell et al. } 1996 \\
\text { Reduce gait } \\
\text { asymmetries of TT } \\
\text { amputees via } \\
\text { real-time visual } \\
\text { feedback }\end{array}$ & $\begin{array}{l}\text { 6 H } \\
\text { Age: } 33-54 \text { yrs (avg. } 42.7 \\
\text { yrs); } \\
6 \text { TT } \\
\text { Cause: } \\
\text { trauma/cancer/peripheral } \\
\text { vascular disease } \\
\text { Age: } 31-69 \text { yrs (avg. } 41.7 \\
\text { yrs) } \\
\text { PE: } 6 \text { mos.-21 yrs (avg. } 6 \\
\text { yrs) } \\
\text { TSA: N/D }\end{array}$ & $\begin{array}{l}\text { FM: Visual } \\
\text { FD: Computer } \\
\text { monitor } \\
\text { FS: Concurrent } \\
\text { S/T: Instrumented } \\
\text { treadmill with force } \\
\text { plates }\end{array}$ & $\begin{array}{l}\text { Lab, } \\
\text { Treadmill }\end{array}$ & $\begin{array}{l}\text { Centre of pressure } \\
(\mathrm{CoP}), \text { stance time } \\
(\%), \text { push off forces, } \\
\text { symmetry index, } \\
\text { single support time }\end{array}$ & N/A & N/A & $\begin{array}{l}4 \text { min of acclimation } \\
\text { (no feedback), } 5 \mathrm{~min} \\
\text { of training with each } \\
\text { feedback parameter } \\
\text { (CoP, stance time } \\
\text { percentage, and } \\
\text { symmetry index), SS } \\
\text { speed }\end{array}$ & $\begin{array}{l}\text { Asymmetrical gait } \\
\text { patterns were } \\
\text { significantly reduced } \\
\text { after providing visual } \\
\text { feedback }\end{array}$ \\
\hline
\end{tabular}


Table 5. Cont

\begin{tabular}{|c|c|c|c|c|c|c|c|c|}
\hline \multirow[b]{2}{*}{$\begin{array}{c}\text { Study } \\
\text { Characteristics }\end{array}$} & \multirow[b]{2}{*}{$\begin{array}{c}\text { Participant's } \\
\text { Characteristics }\end{array}$} & \multirow[b]{2}{*}{$\begin{array}{l}\text { Biofeedback (BFB) } \\
\text { Design }\end{array}$} & \multirow[b]{2}{*}{ Testing Conditions } & \multicolumn{3}{|c|}{ Outcome Measures } & \multirow[b]{2}{*}{$\begin{array}{c}\text { Intervention } \\
\text { Protocol Summary }\end{array}$} & \multirow[b]{2}{*}{ Key Findings } \\
\hline & & & & Gait Parameters & $\begin{array}{c}\text { Physical, } \\
\text { Physiological and } \\
\text { Other Parameters }\end{array}$ & Questionnaire & & \\
\hline $\begin{array}{l}\text { [83] } \\
\text { Sabolich et al. } 1994 \\
\text { Improve balance and } \\
\text { gait by restoring } \\
\text { sensory perception at } \\
\text { the residual } \\
\text { limb/socket interface } \\
\text { via transcutaneous } \\
\text { electrical neural } \\
\text { stimulation }\end{array}$ & $\begin{array}{l}12 \mathrm{TF}, 12 \mathrm{TT} \\
\text { Cause: } \\
\text { trauma/cancer/dysvascular } \\
\text { disease/infection } \\
\text { Age: } 21-68 \text { yrs }(39.5 \pm 13.3 \\
\text { yrs) } \\
\text { PE: } 3 \text { mos.-31 yrs }(8.1 \pm 9.4 \\
\text { yrs) } \\
\text { TSA: N/D }\end{array}$ & $\begin{array}{l}\text { FM: Electrical neural } \\
\text { stimulation } \\
\text { FD: Transcutaneous } \\
\text { electrodes } \\
\text { FS: Concurrent } \\
\text { S/T: Pressure } \\
\text { transducers }\end{array}$ & $\begin{array}{l}\text { Lab, } \\
\text { N/D }\end{array}$ & $\begin{array}{l}\text { Symmetry of weight } \\
\text { distribution, single } \\
\text { limb standing balance, } \\
\text { step length symmetry, } \\
\text { stance time symmetry }\end{array}$ & N/A & N/A & $\begin{array}{l}5 \mathrm{~h} \text { to } 6 \mathrm{~h} \text { walking } \\
\text { with/without } \\
\text { feedback }(10 \mathrm{~min} \\
\text { intervals per } 20 \mathrm{~min} \\
\text { rest) }\end{array}$ & $\begin{array}{l}\text { Both populations } \\
\text { increased weight } \\
\text { distribution } \\
\text { symmetry, step length } \\
\text { symmetry. } \\
\text { Stance time symmetry } \\
\text { and standing balance } \\
\text { improved mainly for } \\
\text { TF amputees. }\end{array}$ \\
\hline $\begin{array}{l}{[17]} \\
\text { Flowers et al. 1986 } \\
\text { Develop and evaluate } \\
\text { a BFB system to } \\
\text { improve prosthetic } \\
\text { weight-bearing and } \\
\text { hip extension }\end{array}$ & $\begin{array}{l}5 \text { TF } \\
\text { Cause: N/D } \\
\text { Age: } 19-68 \text { yrs } \\
\text { PE: N/D } \\
\text { TSA: N/D }\end{array}$ & $\begin{array}{l}\text { FM: Auditory } \\
\text { FD: Earphones or } \\
\text { BFB speakers } \\
\text { FS: Concurrent } \\
\text { S/T: Load cell (or } \\
\text { weight bearing } \\
\text { transducers), } \\
\text { goniometer }\end{array}$ & $\begin{array}{l}\text { Lab, } \\
\text { Overground }\end{array}$ & $\begin{array}{l}\text { Weight bearing, hip } \\
\text { extension angle, steps } \\
\text { count }\end{array}$ & $\mathrm{N} / \mathrm{A}$ & $\mathrm{N} / \mathrm{A}$ & $\begin{array}{l}30 \mathrm{~min} \text { to } 1 \mathrm{~h} \text { sessions } \\
\text { over } 4 \text { months (BFB } \\
\text { device used during } \\
\text { PT sessions) }\end{array}$ & $\begin{array}{l}\text { Subjects with } \\
\text { diminished } \\
\text { awareness of their } \\
\text { bodies and reduced } \\
\text { strength benefited } \\
\text { more from feedback. } \\
\text { BFB improved hip } \\
\text { extension and flexion } \\
\text { at the beginning of } \\
\text { stance phase }\end{array}$ \\
\hline $\begin{array}{l}{[92]} \\
\text { Clippinger et al. } \\
\mathbf{1 9 8 2} \\
\text { Enhance sensory } \\
\text { feedback after lower } \\
\text { limb amputation by } \\
\text { providing electrical } \\
\text { stimulation }\end{array}$ & $\begin{array}{l}13 \text { LLA } \\
(5 \text { Hip disarticulation, } 7 \text { TF, } \\
1 \text { bilateral TT) } \\
\text { Cause: N/D } \\
\text { Age: } \text { N/D } \\
\text { PE: } \text { /D } \\
\text { TSA: } 3 \text { days-4 yrs }\end{array}$ & $\begin{array}{l}\text { FM: Afferent sensory } \\
\text { feedback } \\
\text { FD: Surgically } \\
\text { implanted electrodes } \\
\text { FS: Concurrent } \\
\text { S/T: Piezoelectric } \\
\text { crystal, strain gauges }\end{array}$ & $\begin{array}{l}\mathrm{N} / \mathrm{D}, \\
\mathrm{N} / \mathrm{D}\end{array}$ & Weight bearing & $\mathrm{N} / \mathrm{A}$ & $\mathrm{N} / \mathrm{A}$ & $\begin{array}{l}3 \mathrm{~h} \text { to } 12 \mathrm{~h} \text { of daily } \\
\text { stimulation ranging } \\
\text { from } 8 \text { months to } 6 \\
\text { years }\end{array}$ & $\begin{array}{l}\text { Implanted electrodes } \\
\text { were tolerated by all } \\
\text { patients without } \\
\text { discomfort. } \\
\text { Postoperative pain } \\
\text { reduced and stump } \\
\text { healing improved by } \\
\text { stimulating the sciatic } \\
\text { nerve }\end{array}$ \\
\hline $\begin{array}{l}\text { [93] } \\
\text { Gapsis et al. } 1982 \\
\text { Evaluate a limb load } \\
\text { monitor for } \\
\text { controlling weight } \\
\text { bearing of lower-limb } \\
\text { amputees }\end{array}$ & $\begin{array}{l}20 \text { LLA } \\
(\mathrm{n}=10 \text { study, } \mathrm{n}=10 \text { control } \\
\text { group) } \\
\text { Cause: ND } \\
\text { Age: } 47-78 \text { yrs (avg. } 62.5 \\
\text { yrs) } \\
\text { PE: N/D } \\
\text { TSA: N/D }\end{array}$ & $\begin{array}{l}\text { FM: Auditory } \\
\text { FD: BFB buzzer } \\
\text { FS: Concurrent } \\
\text { S/T: Load sensitive } \\
\text { transducer }\end{array}$ & $\begin{array}{l}\text { Lab, } \\
\text { Overground }\end{array}$ & $\begin{array}{l}\text { Weight bearing } \\
\text { (prosthetic limb load) }\end{array}$ & Total body weight & $\mathrm{N} / \mathrm{A}$ & $\begin{array}{l}5 \text { min for acclimation } \\
\text { period, feedback } \\
\text { system used during } \\
\text { patient's daily } \\
\text { ambulation therapy }\end{array}$ & $\begin{array}{l}\text { Control and study } \\
\text { group reached goals. } \\
\text { Study group reached } \\
\text { goals twice as fast } \\
\text { with feedback }\end{array}$ \\
\hline
\end{tabular}


Table 5. Cont

\begin{tabular}{|c|c|c|c|c|c|c|c|c|}
\hline \multirow[b]{2}{*}{$\begin{array}{c}\text { Study } \\
\text { Characteristics }\end{array}$} & \multirow[b]{2}{*}{$\begin{array}{c}\text { Participant's } \\
\text { Characteristics }\end{array}$} & \multirow[b]{2}{*}{$\begin{array}{c}\text { Biofeedback (BFB) } \\
\text { Design }\end{array}$} & \multirow[b]{2}{*}{ Testing Conditions } & \multicolumn{3}{|c|}{ Outcome Measures } & \multirow[b]{2}{*}{$\begin{array}{c}\text { Intervention } \\
\text { Protocol Summary }\end{array}$} & \multirow[b]{2}{*}{ Key Findings } \\
\hline & & & & Gait Parameters & $\begin{array}{c}\text { Physical, } \\
\text { Physiological and } \\
\text { Other Parameters }\end{array}$ & Questionnaire & & \\
\hline $\begin{array}{l}\text { [85] } \\
\text { Fernie et al. } 1978 \\
\text { BFB device designed } \\
\text { to promote knee } \\
\text { extension at stance } \\
\text { phase }\end{array}$ & $\begin{array}{l}19 \mathrm{TF} \\
\text { Cause: ND } \\
\text { Age: } 46-84 \text { yrs (avg. } 70 \text { yrs) } \\
\text { PE: N/D } \\
\text { TSA: N/D }\end{array}$ & $\begin{array}{l}\text { FM: Auditory } \\
\text { FD: BFB buzzer } \\
\text { FS: Concurrent } \\
\text { S/T: Foot and knee } \\
\text { switch }\end{array}$ & $\begin{array}{l}\mathrm{N} / \mathrm{D}, \\
\mathrm{N} / \mathrm{D}\end{array}$ & $\begin{array}{l}\text { Knee } \\
\text { flexion/extension } \\
\text { angle, steps count }\end{array}$ & $\begin{array}{l}\text { Percentage of errors } \\
\text { (i.e., bending the knee } \\
\text { and loading the limb } \\
\text { simultaneously) }\end{array}$ & $\mathrm{N} / \mathrm{A}$ & $\begin{array}{l}3 \text { weeks of training. } \\
\text { PT involved at early } \\
\text { BFB stages. }\end{array}$ & $\begin{array}{l}\text { Feedback system } \\
\text { encouraged knee } \\
\text { flexion than knee } \\
\text { extension. } \\
\text { Audio signal too } \\
\text { annoying to use.One } \\
\text { participant showed a } \\
\text { period of retention in } \\
\text { the 3rd week of } \\
\text { training }\end{array}$ \\
\hline $\begin{array}{l}\text { [94] } \\
\text { Zimnicki et al. } 1976 \\
\text { BFB system } \\
\text { developed for } \\
\text { geriatric above-knee } \\
\text { amputees to achieve } \\
\text { an adequate knee } \\
\text { extension during } \\
\text { walking }\end{array}$ & $\begin{array}{l}13 \mathrm{TF} \\
\text { Cause: N/D } \\
\text { Age: } 53-84 \text { yrs (avg. } 72 \text { yrs) } \\
\text { PE: N/D } \\
\text { TSA: N/D }\end{array}$ & $\begin{array}{l}\text { FM: Auditory } \\
\text { FD: BFB buzzer } \\
\text { FS: Concurrent } \\
\text { S/T: Pylon switch }\end{array}$ & $\begin{array}{l}\mathrm{N} / \mathrm{D}, \\
\mathrm{N} / \mathrm{D}\end{array}$ & $\begin{array}{l}\text { Knee } \\
\text { flexion/extension } \\
\text { angle, body weight } \\
\text { pressure }\end{array}$ & N/A & N/A & $\begin{array}{l}5 \text { progressive training } \\
\text { stages over } 5 \text { or more } \\
\text { sessions. } \\
\text { PT involved to } \\
\text { reinforce BFB training }\end{array}$ & $\begin{array}{l}\text { BFB found to be more } \\
\text { helpful for } \\
\text { participants who had } \\
\text { difficulty in following } \\
\text { or concentrating on } \\
\text { verbal instructions } \\
\text { and for those one } \\
\text { who appeared to } \\
\text { understand but were } \\
\text { enabled to elicit the } \\
\text { appropriate motor } \\
\text { responses }\end{array}$ \\
\hline $\begin{array}{l}\text { [95] } \\
\text { Warren et al. } 1975 \\
\text { Evaluate the } \\
\text { effectiveness of a BFB } \\
\text { system in comparison } \\
\text { to a Bathroom scale to } \\
\text { improve } \\
\text { weight-bearing }\end{array}$ & $\begin{array}{l}10 \mathrm{H} \\
\text { Cause: N/A } \\
\text { Age: } 18-26 \text { yrs } \\
\text { PE: N/A } \\
\text { TSA: N/A }\end{array}$ & $\begin{array}{l}\text { FM: Auditory, Visual } \\
\text { FD: BFB alarm } \\
\text { FS: Concurrent } \\
\text { S/T: Force plates, } \\
\text { pressure sensitive } \\
\text { insoles }\end{array}$ & $\begin{array}{l}\text { Lab, } \\
\text { Overground }\end{array}$ & Weight-bearing & $\mathrm{N} / \mathrm{A}$ & $\mathrm{N} / \mathrm{A}$ & $\begin{array}{l}\text { Bathroom scale: two } \\
\text { times - four steps on } \\
\text { monitored leg. Three } \\
\text { training levels } \\
\text { with/without } \\
\text { feedback (trying to } \\
\text { reproduce target } \\
\text { loading threshold) }\end{array}$ & $\begin{array}{l}\text { BFB training was of } \\
\text { limited value due to } \\
\text { time lag between } \\
\text { feedback and motor } \\
\text { response }\end{array}$ \\
\hline
\end{tabular}

H: healthy subject; TT: transtibial (below-knee) amputation; TF: transfemoral (above-knee) amputation; PE: prosthetic experience; TSA: time since amputation; FM: feedback modality; FD: feedback device; FS: feedback strategy; S/T: sensors/transducers; SS: self-selected; PT: physiotherapist; N/A: not applicable; N/D: not described. 


\section{Discussion}

The primary aim of this systematic review was to consolidate published evidence that was related to the development and testing of BFB systems as a gait retraining tool for individuals with LLA. The quality of the identified studies $(n=31)$ was generally high, particularly in more recent years (i.e., since 1990).

\subsection{Sample Size}

The average sample size across studies was $13 \pm 3$ non-amputee subjects, $7 \pm 2$ individuals with transtibial amputation, and $3 \pm 1$ individuals with transfemoral amputation. A few studies indicated that statistical tests could not be accurately performed and the findings cannot be extrapolated to larger populations due to their low sample size $[56,58,96]$. It was indicated that, although a small sample limits generalization of findings, a case study could provide pilot data and allow for exploratory research across a diverse population [97,98].

\subsection{User Demographicss}

The majority of lower limb amputees are over 50 years of age and most of the amputations are due to complications that are associated with vascular diseases $[99,100]$. The age of participants with LLA in this review ranged from young adults (as young as 19 years) to the elderly (aged 60 years and above). None of the studies focused on children and youth; a population that might benefit most due to a lifetime of prosthetic use and savviness for technology. There exist limitations that are associated with the transferability of findings between young and older populations. One reason for this is the differences in ambulation ability and the capacity to regain mobility function [100]. Elderly patients may require longer practice time, yet they typically suffer from lower physical endurance [17]. Further, with an older population come challenges with BFB usability. A recent study showed that, in contrast to healthy young adults, elderly healthy subjects were unable to utilize BFB information to reduce trunk sway while walking distracted [101]. Walking can be considered to be an unconscious (i.e., low cognitive) activity for healthy subjects [102]. However, for prosthetic users, walking often increases cognitive load and energy expenditure $[103,104]$. Prosthetic users usually depend on additional information (i.e., visual, auditory, and somatosensory) to ambulate safety [10].

Phantom limb pain affects many individuals with lower-limb amputation. It is manifested as sensations or pain from a body part that no longer exists [105]. Techniques, such as mirror therapy and BFB, have demonstrated the potential to reduce phantom limb pain in prosthetic users $[59,86,105]$. An important benefit of BFB systems when compared to mirror therapy approaches is that, in addition to reducing phantom pain, BFB systems can potentially improve the overall gait performance and prosthetic function of individuals with LLA $[59,86]$. Accordingly, participant characteristics (i.e., age, etiology, level of amputation, and prosthetic experience) should be considered in the development an effective and user-friendly BFB system, as these may dictate the most suitable BFB modalities (i.e., visual, auditory, or haptic feedback) and BFB strategies (i.e., control algorithms utilized to convey sensory feedback to BFB users).

\subsection{Level of Amputation}

Transfemoral amputees typically demonstrate more severe gait deviations than transtibial amputees [106]. There is also increased energy cost, loss of mobility function and decrease in walking efficiency with higher levels of amputation [97]. However, transfemoral amputees are generally underrepresented in the BFB research. Additionally, the targeted gait parameters appear to be dependent on the specific amputee demographic. For instance, gait symmetry ratios, stance times, hip and knee flexion/extension angles, and trunk sway are more relevant for transfemoral than those for transtibial amputees [6,107]. One study found it difficult to recruit transtibial amputees with asymmetric gait and recommended recruiting transfemoral amputees [36]. On the other hand, the gait 
of transfemoral amputees and their ability to make gait adjustments is highly dependent on the function of the prosthesis, and particularly the prosthetic knee joint [56]. In one study, for example, because of the inability of the artificial knee-joint to actively adjust, the TF amputee could only vary gait speed with the healthy limb [108]. Hence, improvements in gait may require gait retraining, but also adjustments to the prosthetic setup, particularly with higher-level amputations. While this might become less of an issue with self-adjusting microprocessor knee joints, it will be imperative that the BFB and prosthetic control systems are designed to work symbiotically. It was also suggested that future research should determine how different amputee populations can benefit from different $B F B$ modalities and different BFB strategies $[28,56,88]$.

\subsection{Prosthetic Experience and Time Since Amputation}

The best practices in amputee rehabilitation encourage physiotherapy, prosthetic fitting, and training to be provided as soon as possible post amputation. One study involving established prosthetic users ( $>2$ years post amputation) suggested that research should focus on how effective BFB is in the early stages of rehabilitation [56]. Others suggest that the best results occur with novice users, but that experiments should be done on expert prosthesis users to examine effectiveness [28]. To this point, one study found that newer, less established amputees were better able to adapt their gait patterns [36]. While early rehabilitation using BFB has been suggested for other populations, such as stroke, this approach presents certain challenges in amputee research and rehabilitation [109]. Issues, such as prosthetic fit and residuum healing can complicate experimentation on new amputees [97]. Overloading in the post-operation stage might result in tissue breakdown [90] and premature rehabilitation might affect the incision and cause healing issues [110]. BFB systems have been developed to improve the healing process in the early postoperative period by warning amputees applying excessive pressure on the residual limb [90]. Several studies had confounded results, since the patients were being provided conventional physiotherapy during the time of the BFB experiments; this is a potential issue when testing with recent amputees receiving standard care $[81,86,97]$. Similarly, to limit the confounding effects, one study withheld prosthetic alignment changes as the participants began to exhibit better gait patterns. However, this goes against standard care and might have contributed to poorer results in the study [81]. Based on these findings, BFB training should be provided as soon as the residuum is healed and volume stabilized, a satisfactory and stable prosthetic setup, alignment and fit have been achieved, and conventional physiotherapy treatments have concluded to exclusively assess the effectiveness of the BFB systems.

\subsection{BFB Intervention (Experimental Protocol)}

None of the studies tested BFB under a randomized control trial (RCT). RCTs minimise the risk of confounding factors that might alter the results. For this reason, RCT studies are the golden standard for validating the effectiveness of an intervention. Over one-quarter of the studies reported using a single gait training session. It is important to allow for the user to have adequate training with the BFB system to enhance learning [64]. Training is important, as making errors drives motor learning [39]. Studies often do not report instructions given or training methods and research should be done to determine the best method of conducting the training sessions [111]. The effects of training intensity were mentioned in a few of the included papers and conclusions were mixed. One study concluded that outcomes are better with more intensive training [56], while others suggest gradually setting attainable targets [88]. Moreover, as in previous reviews, most studies did not report any follow-up sessions with the BFB system to test for retention $[22,64]$. Finally, the literature is unclear as to how BFB should be integrated into conventional physiotherapy. For example, systems, such as the one described by Redd et al., can be used with little specialized training and without the supervision of a physiotherapist/prosthetists [88]. It is likely that BFB might be most effective in combination with existing physiotherapy and gait training practices [112]. 


\subsection{Treadmill vs. Overground Walking}

Most studies used a treadmill during the experimental procedure. Nagano et al. has shown that temporal gait parameters, such as double stance time and swing time differ when walking on a treadmill compare than those walking overground [101]. Another study showed that walking on a treadmill reduces dorsiflexor and knee extensor moments [102] and increases hip extensor moments in the sagittal plane [102]. This suggests that BFB strategies utilized to alter gait symmetry might need to be modified, depending on treadmill or overground walking. Further, it has been shown that difficulty might arise when translating locomotor skills from treadmill training to overground walking [103].

\subsection{BFB Parameter Measurements}

Only some of the studies validated the BFB system's accuracy in sensing the targeted gait parameter(s). For example, Isakov et al. validated their pressure sensing insoles with a force plate [66] and Yang et al. used a previously validated BFB system consisting of a force plate and motion analysis system [36,113]. The inaccuracy of a goniometer for knee angle measurements was a limitation in one study [87]. Another study noted a source of error in the algorithm to detect heel-strike and toe-off [88]. The accurate and reliable measurement of gait deviations is crucial to the success of wearable BFB systems, as erroneous feedback and false positives calling for corrections in gait can confuse and frustrate BFB users. Few studies mentioned time delays in their BFB system $[30,87,114]$. For instance, Crea et al. [30] and Liu et al. [114] reported delays less than or equal to 200ms due to wireless communication with the sensors embedded in the shoe-insoles. Consequently, delays were produced in the detection of the feedback stimulus. Accordingly, effective BFB systems must have low latency, especially when sensing and providing real-time feedback during dynamic activities, such as walking.

\subsection{BFB Modality}

Visual feedback was the most common method provided to the participants, but there is some debate on the most appropriate and effective feedback modality. In one study, visual feedback was deemed the most intuitive modality that was based on user preference and usability [88]; however, the authors suggested that more work should be done to improve the usability/ease of use of vibrotactile and auditory feedback [88]. One study found that amputees and physiotherapists valued auditory over visual feedback and the participants adapted more easily to auditory feedback [56]. These preferences appear to be related to BFB design, testing, and safety aspects. BFB users might prefer or find more useful the BFB modality that provides more intuitive and relevant feedback information according to the gait parameter and task performed. For example, visual feedback is typically confined to specific locations (e.g., treadmill walking while watching a display under laboratory conditions). Safety issues relating to falling can arise during activities of daily living, for example, as prosthetic users walk and negotiate obstacles, such as curbs and stairs while watching a display or a smartphone. For this reason, auditory and haptic (e.g., vibrotactile) feedback may be more suitable for field or community-based systems [64]. A previous mapping review came to similar conclusions. It noted that, while visual feedback was most commonly used and studied, it might not be the most effective feedback modality for practical use [64]. Visual feedback is more appropriate for the perception of spatial information [115], while auditory and haptic feedback are better suited for the perception of temporal [115] and spatiotemporal information [39,115], respectively, according to the literature in motor learning. Accordingly, BFB systems need to appropriately align feedback modalities and strategies with measured gait parameters. For instance, in terms of vibrotactile feedback, diverse feedback strategies (i.e., a combination of vibration patterns-vibration levels and activation sequences-including different locations and number of vibrating motors) have been utilized to improve gait performance $[28,36,40,58,88,116]$. However, a systematic comparison of the current 
implemented feedback strategies is missing to explore which strategy might produce greater positive gait outcomes on individuals with LLA.

Of the reviewed studies, six $(n=6)$ provided multimodal feedback $[58,74-76,88]$. Some researchers have suggested that multimodal feedback reduces cognitive load and can enhance motor learning $[39,58,83]$. For instance, Crea et al. evaluated the cognitive load of a visual-vibrotactile BFB system by adding a secondary cognitive task (i.e., serial subtraction) during walking with vibrotactile feedback [58]. The results showed that gait symmetry was improved without significant increases on cognitive load in the presence of feedback walking [58]. In another study, Pagel et al. showed that, when cognitive load increases, the imbalance between intact and prosthetic limb becomes more pronounced [84]. Cognitive impairment appears to be more common in individuals with LLA than in the general population-this is linked to difficulties with regaining mobility and independence after amputation [117]. Thus, if the feedback is too cognitively taxing, it might be counterproductive and more difficult for amputees to process the information, potentially even resulting in worsened gait and mobility performance [112]. For instance, Fernie et al. [85] designed an auditory BFB system to maintain knee extension through the stance phase; however, the BFB system was found to alter knee flexion instead. Chow et al. [90] originally designed an auditory BFB to encourage participants to increase the loading of the residual limb, but in fact the audio BFB prevented adequate loading of the residual limb.

\subsection{Feedback Strategies}

Feedback strategies mainly utilized baseline (no feedback) conditions to obtain an initial value of the specific gait parameters. Most of the studies set a gait target threshold for participants prior to BFB testing. Few studies set these thresholds based on the feedback provided by a physiotherapist, who assessed the participant's walking ability to personalize the BFB treatment or provided verbal cues prior or during the early stages of BFB gait retraining $[17,34,36,81,85,94]$. Feedback can be provided in real-time (concurrent feedback) or after the trial has finished (terminal feedback) [112]. All the studies applied concurrent feedback, and none provided terminal feedback. A recent review [112] showed that concurrent feedback produces the best short-term results, while terminal feedback produces the best long-term results [112]. Therefore, an effective training strategy could be to provide concurrent $\mathrm{BFB}$ and terminal feedback from the physiotherapist during training sessions.

\subsection{Other BFB design and Application Considerations}

When designing a BFB system, it is important that the BFB strategy is non-obtrusive and enjoyable for the user. Program adherence has been linked to program enjoyment [97]. This will ensure that the BFB systems will be used in the long-term. Motivation for walking is a predictive factor for successful rehabilitation of amputees [118]. If a wearable daily use system is not the goal for the researcher, virtual reality (VR) systems may be a good option to motivate and encourage program adherence, since users might perceive to have more interaction with the system. For balance, the Wii-Fit has been shown to improve the balance and gait in older adults [97], children and adolescents with amputation [119], as well as individuals with Alzheimer's and Parkinson's disease [120]. Although the Wii-Fit is no longer commercially available, other VR options have been used for rehabilitation purposes, including the CAREN system and C-Mill (both from Motekforce Link, Amsterdam). The two included studies used VR environments, specifically the CAREN system [34,81]. Virtual reality might be the best option for BFB because program adherence is important [97]. Alternately, if BFB systems are envisioned as wearable systems that are built into prostheses that provide as needed feedback during activities of daily living, they must do so unobtrusively and seamlessly. However, to date, only two studies tested their systems in the field $[59,86]$. Moreover, retention and fading, which are important considerations for the continuous use of feedback systems, are not well understood and require further attention [64]. 


\subsection{Limitations of the Systematic Review}

It was not possible to conduct a meta-analysis on the data due to the wide range of target gait parameters, outcome measures, and methods used. Further, six databases as well as the references from included studies were used to search for articles. Articles that were not written in English were not included.

\section{Conclusions}

Although most individuals with amputation are older, there is a lack of research on technology-based feedback for a younger or paediatric population. Further, the older population might have difficulty with the usability and response times of BFB systems. Different amputation levels may benefit from different feedback strategies and/or target parameters; therefore, it is important to investigate the effect on gait of different feedback strategies to ensure that the target gait parameter and the sensory information are appropriate for the target population. BFB training should be provided as soon as possible in the rehabilitation stage, but the training should not start until the stabilization of the early stages of the rehabilitation process. Auditory and vibrotactile feedback are more wearable and different population ages may respond to feedback differently, and it is important to align feedback modality and feedback strategies appropriately with the measured gait parameter(s). The relationship between training intensity and performance is unknown and future work should be conducted to investigate a possible correlation.

In terms of developing an effective, robust, and user-friendly wearable biofeedback system to improve the gait of individuals with LLA, the following aspects should be considered: (1) target gait parameters should be clinically relevant for the targeted population. For instance, gait symmetry ratios, stance times, hip and knee flexion/extension angles, and trunk sway are more relevant for transfemoral than those for transtibial amputees; (2) BFB modalities (i.e., visual, auditory, haptic, and multimodal feedback) should take into consideration usage conditions (i.e., laboratory, clinical, or home-care settings), including user's age, level of amputation, and prosthetic experience; (3) feedback information (i.e., BFB strategies) should be easy to perceive, discriminate, and utilize by BFB users, allowing for them to transfer feedback information with low cognitive demand; (4) wearable sensors, such as pressure sensors, load cells, electrogoniometers, etc. should be fully integrated into BFB systems to improve wearability. In addition, accelerometers, gyroscopes, or inertial measurement units (IMU sensors) are encouraged to be used for gait event detection, which might improve accuracy and portability of the BFB systems; and, (5) program adherence and program enjoyment should be sought to ensure the long-term use of BFB systems. Effective BFB systems might be achieved by designing a goal-oriented experimental intervention and by considering the previously mentioned points regarding BFB design.

Supplementary Materials: The following are available online http://www.mdpi.com/1424-8220/20/6/1628/s1. The PRISMA Checklist utilized to conduct the proposed systematic review is included as a supplementary material.

Author Contributions: Conceptualization, R.E., A.M. and J.A.; methodology, R.E.-N., A.M. and J.A.; validation, R.E.-N., A.M. and J.A.; formal analysis, R.E.-N., A.M. and J.A.; investigation, R.E.-N., A.M. and J.A.; writing-original draft preparation, R.E.-N., A.M. and J.A.; writing-review and editing, R.E.-N., A.M. and J.A.; visualization, R.E.-N., A.M. and J.A.; supervision, J.A.; funding acquisition, J.A. All authors have read and agreed to the published version of the manuscript.

Funding: This research was funded by Natural Sciences and Engineering Research Council of Canada (NSERC) Discovery RGPIN 2018-05046, Mexico's National Council for Science and Technology (CONACyT), and Ontario Council on Graduate Studies, Council of Ontario Universities (Ontario Graduate Scholarship).

Acknowledgments: The authors would like to thank Pui-Ying Wong for her support as an experienced librarian during the search strategy process. And, to Firdous Hadj-Moussa for her support in reviewing and editing the manuscript.

Conflicts of Interest: The authors declare no conflict of interest. The funders had no role in the design of the study; in the collection, analyses, or interpretation of data; in the writing of the manuscript, or in the decision to publish the results. 


\section{References}

1. Latanioti, E.P.; Angoules, A.; Boutsikari, E.C. Proprioception in Above-the-Knee Amputees with Artificial Limbs. Sci. World J. 2013, 2013, 1-5. [CrossRef] [PubMed]

2. A Nurse, M.; Nigg, B. The effect of changes in foot sensation on plantar pressure and muscle activity. Clin. Biomech. 2001, 16, 719-727. [CrossRef]

3. Adams, J.A.; Dickinson, J. Proprioceptive Control of Human Movement. Am. J. Psychol. 1977, 90, 336. [CrossRef]

4. Geurts, A.C.H.; Mulder, T.W. Reorganisation of Postural Control Following Lower Limb Amputation: Theoretical Considerations and Implications for Rehabilitation. Physiother. Theory Pr. 1992, 8, 145-157. [CrossRef]

5. Jaegers, S.M.; Arendzen, J.H.; De Jongh, H.J. Prosthetic gait of unilateral transfemoral amputees: A kinematic study. Arch. Phys. Med. Rehabil. 1995, 76, 736-743. [CrossRef]

6. Nolan, L.; Wit, A.; Dudziñski, K.; Lees, A.; Lake, M.; Wychowañski, M. Adjustments in gait symmetry with walking speed in trans-femoral and trans-tibial amputees. Gait Posture 2003, 17, 142-151. [CrossRef]

7. Hermodsson, Y.; Ekdahl, C.; Persson, B.M.; Roxendal, G. Standing balance in trans-tibial amputees following vascular disease or trauma: A comparative study with healthy subjects. Prosthet. Orthot. Int. 1994, 18, 150-158. [CrossRef]

8. Gailey, R. Review of secondary physical conditions associated with lower-limb amputation and long-term prosthesis use. J. Rehabil. Res. Dev. 2008, 45, 15-30. [CrossRef]

9. Miller, W.C.; Speechley, M.; Deathe, B. The prevalence and risk factors of falling and fear of falling among lower extremity amputees. Arch. Phys. Med. Rehabil. 2001, 82, 1031-1037. [CrossRef]

10. Miller, W.C.; Deathe, A.; Speechley, M.; Koval, J.J. The influence of falling, fear of falling, and balance confidence on prosthetic mobility and social activity among individuals with a lower extremity amputation. Arch. Phys. Med. Rehabil. 2001, 82, 1238-1244. [CrossRef]

11. Kulkarni, J.; Wright, S.; Toole, C.; Morris, J.; Hirons, R. Falls in Patients with Lower Limb Amputations: Prevalence and Contributing Factors. Physiotherapy 1996, 82, 130-136. [CrossRef]

12. Hunter, S.W.; Batchelor, F.; Hill, K.; Hill, A.-M.; Mackintosh, S.; Payne, M. Risk Factors for Falls in People With a Lower Limb Amputation: A Systematic Review. PMER 2016, 9, 170-180.

13. A Behar, T.; Burnham, S.J.; Johnson, G. Major stump trauma following below-knee amputation. Outcome and recommendations for therapy. J. Cardiovasc. Surg. 1991, 32, 753-756.

14. Miller, W.C.; Speechley, M.; Deathe, A.B. Balance Confidence among People with Lower-Limb Amputations. Phys. Ther. 2002, 82, 856-865. [CrossRef]

15. Gauthier-Gagnon, C.; Grisé, M.-C.; Potvin, D. Enabling factors related to prosthetic use by people with transtibial and transfemoral amputation. Arch. Phys. Med. Rehabil. 1999, 80, 706-713. [CrossRef]

16. Williamson, G.M.; Schulz, R.; Bridges, M.W.; Behan, A.M. Social and psychological factors in adjustment to limb amputation. J. Soc. Behav. Pers. 1994, 9, 249.

17. Flowers, W.C.; Cullen, C.P.; Tyra, K.P. A preliminary report on the use of a practical biofeedback device for gait training of above-knee amputees. J. Rehabil. Res. Dev. 1986, 23, 7-18.

18. Broomhead, P.; Dawes, D.; Hale, C.; Lambert, A.; Quinlivan, D.; Shepherd, R. Evidence based Clinical Guidelines for the Physiotherapy Management of Adults with Lower Limb Prostheses; British Association of Chartered Physiotherapists in Amputation Rehabilitation: London, UK, 2003.

19. Sjödahl, G.-B.J.C. Gait Improvement in Unilateral Transfemoral Amputees by a Combined Psychological and Physiotherapeutic Treatment. J. Rehabil. Med. 2001, 33, 114-118.

20. Sjödahl, C.; Jarnlo, G.-B.; Söderberg, B.; Persson, B. Kinematic and kinetic gait analysis in the sagittal plane of trans-femoral amputees before and after special gait re-education. Prosthet. Orthot. Int. 2002, 26, 101-112. [CrossRef]

21. Rau, B.; Bonvin, F.; De Bie, R. Short-term effect of physiotherapy rehabilitation on functional performance of lower limb amputees. Prosthet. Orthot. Int. 2007, 31, 258-270. [CrossRef]

22. Tate, J.J.; Milner, C. Real-Time Kinematic, Temporospatial, and Kinetic Biofeedback During Gait Retraining in Patients: A Systematic Review. Phys. Ther. 2010, 90, 1123-1134. [CrossRef] [PubMed]

23. Staiano, A.E.; Flynn, R. Therapeutic Uses of Active Videogames: A Systematic Review. Games Heal. J. 2014, 3, 351-365. [CrossRef] [PubMed] 
24. Miller, C.A.; Hayes, D.M.; Dye, K.; Johnson, C.; Meyers, J. Using the Nintendo Wii Fit and Body Weight Support to Improve Aerobic Capacity, Balance, Gait Ability, and Fear of Falling. J. Geriatr. Phys. Ther. 2012, 35, 95-104. [CrossRef] [PubMed]

25. Shull, P.B.; Damian, D.D. Haptic wearables as sensory replacement, sensory augmentation and trainer - a review. J. Neuroeng. Rehabil. 2015, 12, 59. [CrossRef]

26. Rusaw, D.F.; Hagberg, K.; Nolan, L.; Ramstrand, N. Can vibratory feedback be used to improve postural stability in persons with transtibial limb loss? J. Rehabil. Res. Dev. 2012, 49, 1239. [CrossRef]

27. Giggins, O.M.; Persson, U.M.; Caulfield, B. Biofeedback in rehabilitation. J. Neuroeng. Rehabil. 2013, 10, 60. [CrossRef]

28. Plauche, A.; Villarreal, D.; Gregg, R. A Haptic Feedback System for Phase-Based Sensory Restoration in Above-Knee Prosthetic Leg Users. IEEE Trans. Haptics 2016, 9, 421-426. [CrossRef]

29. Ma, C.Z.-H.; Wong, D.W.-C.; Lam, W.-K.; Wan, A.H.-P.; Lee, C.C.W. Balance Improvement Effects of Biofeedback Systems with State-of-the-Art Wearable Sensors: A Systematic Review. Sensors 2016, 16, 434. [CrossRef]

30. Crea, S.; Cipriani, C.; Donati, M.; Carrozza, M.C.; Vitiello, N. Providing Time-Discrete Gait Information by Wearable Feedback Apparatus for Lower-Limb Amputees: Usability and Functional Validation. IEEE Trans. Neural Syst. Rehabil. Eng. 2014, 23, 250-257. [CrossRef]

31. Sharma, A.; Torres-Moreno, R.; Zabjek, K.; Andrysek, J. Toward an artificial sensory feedback system for prosthetic mobility rehabilitation: Examination of sensorimotor responses. J. Rehabilitation Res. Dev. 2014, 51, 907-917. [CrossRef]

32. Eshraghi, A.; Safaeepour, Z.; Geil, M.; Andrysek, J. Walking and balance in children and adolescents with lower-limb amputation: A review of literature. Clin. Biomech. 2018, 59, 181-198. [CrossRef] [PubMed]

33. Andrysek, J.; Naumann, S.; Cleghorn, W. Design and Quantitative Evaluation of a Stance-Phase Controlled Prosthetic Knee Joint for Children. IEEE Trans. Neural Syst. Rehabil. Eng. 2005, 13, 437-443. [CrossRef] [PubMed]

34. Esposito, E.R.; Choi, H.S.; Darter, B.J.; Wilken, J. Can real-time visual feedback during gait retraining reduce metabolic demand for individuals with transtibial amputation? PLOS ONE 2017, 12, e0171786.

35. Brandt, A.; Riddick, W.; Stallrich, J.W.; Lewek, M.; Huang, H. (Helen) Effects of extended powered knee prosthesis stance time via visual feedback on gait symmetry of individuals with unilateral amputation: A preliminary study. J. Neuroeng. Rehabil. 2019, 16, 112. [CrossRef]

36. Yang, L.; Dyer, P.; Carson, R.; Webster, J.; Foreman, K.B.; Bamberg, S.J.M. Utilization of a lower extremity ambulatory feedback system to reduce gait asymmetry in transtibial amputation gait. Gait Posture 2012, 36, 631-634. [CrossRef]

37. Afzal, M.R.; Pyo, S.; Oh, M.-K.; Park, Y.S.; Lee, B.-C.; Yoon, J. Haptic based gait rehabilitation system for stroke patients. In Proceedings of the 2016 IEEE/RSJ International Conference on Intelligent Robots and Systems (IROS), Daejeon, Korea, 9-14 October 2016; pp. 3198-3203.

38. Afzal, M.R.; Oh, M.-K.; Choi, H.Y.; Yoon, J. A novel balance training system using multimodal biofeedback. Biomed. Eng. Online 2016, 15, 42. [CrossRef]

39. Sigrist, R.; Rauter, G.; Riener, R.; Wolf, P. Augmented visual, auditory, haptic, and multimodal feedback in motor learning: A review. Psychon. Bull. Rev. 2012, 20, 21-53. [CrossRef]

40. Afzal, M.R.; Pyo, S.; Oh, M.-K.; Park, Y.S.; Yoon, J. Identifying the effects of using integrated haptic feedback for gait rehabilitation of stroke patients. In Proceedings of the 2017 International Conference on Rehabilitation Robotics (ICORR), London, UK, 17-20 July 2017; pp. 1055-1060.

41. Basmajian, J.V.; Kukulka, C.G.; Narayan, M.G.; Takebe, K. Biofeedback treatment of foot-drop after stroke compared with standard rehabilitation technique: effects on voluntary control and strength. Arch. Phys. Med. Rehabil. 1975, 56, 231-236.

42. Glanz, M.; Klawansky, S.; Chalmers, T. Biofeedback therapy in stroke rehabilitation: a review. J. R. Soc. Med. 1997, 90, 33-39. [CrossRef]

43. Ghoseiri, K.; Forogh, B.; Sanjari, M.A.; Bavi, A. The Effect of a Vibratory Lumber Orthosis on Walking Velocity in Patients with Parkinson's Disease. Prosthet. Orthot. Int. 2009, 33, 82-88. [CrossRef]

44. Shen, X.; Mak, M. Balance and Gait Training with Augmented Feedback Improves Balance Confidence in People With Parkinson's Disease. Neurorehabilit. Neural Repair 2014, 28, 524-535. [CrossRef] [PubMed] 
45. El-Tamawy, M.; Darwish, M.; Khallaf, M. Effects of augmented proprioceptive cues on the parameters of gait of individuals with Parkinson's disease. Ann. Indian Acad. Neurol. 2012, 15, 267. [CrossRef] [PubMed]

46. MacIntosh, A.; Lam, E.; Vigneron, V.; Vignais, N.; Biddiss, E. Biofeedback interventions for individuals with cerebral palsy: a systematic review. Disabil. Rehabil. 2019, 41, 2369-2391. [CrossRef] [PubMed]

47. Sienko, K.; Whitney, S.; Carender, W.; Wall, C.; Iii, C.W. The role of sensory augmentation for people with vestibular deficits: Real-time balance aid and/or rehabilitation device? J. Vestib. Res. 2017, 27, 63-76. [CrossRef]

48. Horlings, C.G.C.; Carpenter, M.; Honegger, F.; Allum, J.H.J. Vestibular and Proprioceptive Contributions to Human Balance Corrections. Ann. N. Y. Acad. Sci. 2009, 1164, 1-12. [CrossRef]

49. E Aikens, J. Thermal biofeedback for claudication in diabetes: a literature review and case study. Altern. Med. Rev. 1999, 4, 104-110.

50. Witteveen, H.J.B.; Luft, F.; Rietman, J.S.; Veltink, P. Stiffness Feedback for Myoelectric Forearm Prostheses Using Vibrotactile Stimulation. IEEE Trans. Neural Syst. Rehabilitation Eng. 2013, 22, 53-61. [CrossRef]

51. Antfolk, C.; D'Alonzo, M.; Rosén, B.; Lundborg, G.; Sebelius, F.; Cipriani, C. Sensory feedback in upper limb prosthetics. Expert Rev. Med Devices 2013, 10, 45-54. [CrossRef]

52. Leineweber, M.; Shi, S.; Andrysek, J. A Method for Evaluating Timeliness and Accuracy of Volitional Motor Responses to Vibrotactile Stimuli. J. Vis. Exp. 2016, 114, 1-7. [CrossRef]

53. Sharma, A.; Leineweber, M.J.; Andrysek, J. Effects of cognitive load and prosthetic liner on volitional response times to vibrotactile feedback. J. Rehabil. Res. Dev. 2016, 53, 473-482. [CrossRef]

54. Tino, A.; Carvalho, M.; Preto, N.F.; McConville, K.M.V. Wireless vibrotactile feedback system for postural response improvement. In Proceedings of the 2011 Annual International Conference of the IEEE Engineering in Medicine and Biology Society, Boston, MA, USA, 30 August-3 September 2011; pp. 5203-5206.

55. Alahakone, A.U.; Senanayake, S.M.N.A. A Real-Time System with Assistive Feedback for Postural Control in Rehabilitation. IEEE/ASME Trans. Mechatron. 2010, 15, 226-233. [CrossRef]

56. Lee, M.-Y.; Lin, C.-F.; Soon, K.-S. Balance control enhancement using sub-sensory stimulation and visual-auditory biofeedback strategies for amputee subjects. Prosthet. Orthot. Int. 2007, 31, 342-352. [CrossRef] [PubMed]

57. Fiedler, G.; Kutina, K. Feasibility of a mobile feedback system for gait retraining in people with lower limb loss-A technical note. J. Rehabil. Assist. Technol. Eng. 2019, 6, 205566831881368. [CrossRef]

58. Crea, S.; Edin, B.B.; Knaepen, K.; Meeusen, R.; Vitiello, N. Time-Discrete Vibrotactile Feedback Contributes to Improved Gait Symmetry in Patients With Lower Limb Amputations: Case Series. Phys. Ther. 2017, 97, 198-207. [CrossRef]

59. Petrini, F.M.; Bumbasirevic, M.; Valle, G.; Ilic, V.; Mijović, P.; Čvančara, P.; Barberi, F.; Katic, N.; Bortolotti, D.; Andreu, D.; et al. Sensory feedback restoration in leg amputees improves walking speed, metabolic cost and phantom pain. Nat. Med. 2019, 25, 1356-1363. [CrossRef]

60. Rokhmanova, N.; Rombokas, E. Vibrotactile Feedback Improves Foot Placement Perception on Stairs for Lower-Limb Prosthesis Users. In Proceedings of the 2019 IEEE 16th International Conference on Rehabilitation Robotics (ICORR), Toronto, ON, Canada, 24-28 June 2019; Volume 2019, pp. 1215-1220.

61. Begg, R.; Tirosh, O.; Van Der Straaten, R.; Sparrow, W.A. Real-time biofeedback of gait parameters using infrared position sensors. In Proceedings of the 2012 Sixth International Conference on Sensing Technology (ICST), Kolkata, India, 18-21 December 2012; pp. 73-77.

62. Wan, A.H.; Wong, D.W.-C.; Ma, C.Z.-H.; Zhang, M.; Lee, C.C.W. Wearable Vibrotactile Biofeedback Device Allowing Identification of Different Floor Conditions for Lower-Limb Amputees. Arch. Phys. Med. Rehabil. 2016, 97, 1210-1213. [CrossRef]

63. Shi, S.; Leineweber, M.; Andrysek, J. Exploring the Tactor Configurations of Vibrotactile Feedback Systems for Use in Lower-Limb Prostheses1. J. Vib. Acoust. 2019, 141, 1-38. [CrossRef]

64. Van Gelder, L.; Barnes, A.; Wheat, J.S.; Heller, B. The use of biofeedback for gait retraining: A mapping review. Clin. Biomech. 2018, 59, 159-166. [CrossRef]

65. Zijlstra, A.; Mancini, M.; Chiari, L.; Zijlstra, W. Biofeedback for training balance and mobility tasks in older populations: a systematic review. J. Neuroeng. Rehabil. 2010, 7, 58. [CrossRef]

66. Isakov, E. Gait rehabilitation: a new biofeedback device for monitoring and enhancing weight-bearing over the affected lower limb. Eur. Medicophysica 2006, 43, 21-26. 
67. Hershko, E.; Tauber, C.; Carmeli, E. Biofeedback versus physiotherapy in patients with partial weight-bearing. Am. J. Orthop. 2008, 37, 92-96.

68. Gauthier-Gagnon, C. Augmented sensory feedback in the early training of standing balance of below-knee amputees. Physiother. Can. 1986, 137-142.

69. Verhagen, A.P.; de Vet, H.C.W.; de Bie, R.A.; Kessels, A.G.H.; Boers, M.; Bouter, L.M.; Knipschild, P.G. The Delphi List. J. Clin. Epidemiol. 1998, 51, 1235-1241. [CrossRef]

70. Oxman, A.D. Systematic Reviews: Checklists for review articles. BMJ 1994, 309, 648-651. [CrossRef]

71. Downs, S.H.; Black, N. The feasibility of creating a checklist for the assessment of the methodological quality both of randomised and non-randomised studies of health care interventions. J. Epidemiol. Commun. Heal. 1998, 52, 377-384. [CrossRef]

72. Peters, A.; Galna, B.; Sangeux, M.; Morris, M.; Baker, R. Quantification of soft tissue artifact in lower limb human motion analysis: A systematic review. Gait Posture 2010, 31, 1-8. [CrossRef]

73. Ku, P.X.; Abu Osman, N.A.; Abas, W.A.B.W. Balance control in lower extremity amputees during quiet standing: A systematic review. Gait Posture 2014, 39, 672-682. [CrossRef]

74. Lee, M.-Y.; Soon, K.-S. New foot pressure activated sensory compensation posture-control enhancement in amputee. In Proceedings of the 2008 IEEE International Conference on Systems, Man and Cybernetics (SMC), Suntec, Singapore, 12-15 October 2008; Volume 12, pp. 2566-2571.

75. Lee, M.-Y.; Soon, K.-S.; Lin, C.-F. New Computer Protocol with Subsensory Stimulation and Visual/Auditory Biofeedback for Balance Assessment in Amputees. J. Comput. 2009, 4, 52-59. [CrossRef]

76. Lee, M.-Y.; Soon, K.-S. Subsensory stimulation and visual/auditory biofeedback for balance control in amputees. In Proceedings of the 2010 International Conference on Networking, Sensing and Control (ICNSC), Chicago, IL, USA, 10-12 April 2010; pp. 89-94.

77. Lin, M.-Y.; Lee, M.-Y.; Soon, K.-S.; Lin, W.-Y.; Shieh, W.-Y. A New Biofeedback Lower Limb Neuroprothesis for Balance Control and Sensory Compensation in Amputees. J. Neurosci. Neuroeng. 2013, 2, 90-97. [CrossRef]

78. Brandt, A.; Liu, M.; Huang, H.H. Does the impedance of above-knee powered prostheses need to be adjusted for load-carrying conditions? In Proceedings of the 2016 38th Annual International Conference of the IEEE Engineering in Medicine and Biology Society (EMBC), Orlando, FL, USA, 16-20 August 2016.

79. Davis, B.L.; (Cater) Ortolano, M.; Richards, K.; Redhed, J.; Kuznicki, J.; Sahgal, V. Realtime Visual Feedback Diminishes Energy Consumption of Amputee Subjects during Treadmill Locomotion. JPO J. Prosthet. Orthot. 2004, 16, 49-54. [CrossRef]

80. Petrini, F.M.; Valle, G.; Bumbasirevic, M.; Barberi, F.; Bortolotti, D.; Cvancara, P.; Hiairrassary, A.; Mijovic, P.; Sverrisson, A.Ö.; Pedrocchi, A.; et al. Enhancing functional abilities and cognitive integration of the lower limb prosthesis. Sci. Transl. Med. 2019, 11, eaav8939. [CrossRef] [PubMed]

81. Darter, B.J.; Wilken, J. Gait Training With Virtual Reality-Based Real-Time Feedback: Improving Gait Performance Following Transfemoral Amputation. Phys. Ther. 2011, 91, 1385-1394. [CrossRef] [PubMed]

82. Huang, S.; Wensman, J.P.; Ferris, D.P. Locomotor Adaptation by Transtibial Amputees Walking With an Experimental Powered Prosthesis under Continuous Myoelectric Control. IEEE Trans. Neural Syst. Rehabil. Eng. 2016, 24, 573-581. [CrossRef] [PubMed]

83. Sabolich, J.A.; Ortega, G.M. Sense of Feel for Lower-Limb Amputees: A Phase-One Study. JPO J. Prosthet. Orthot. 1994, 6, 36-41. [CrossRef]

84. Pagel, A.; Arieta, A.H.; Riener, R.; Vallery, H. Effects of sensory augmentation on postural control and gait symmetry of transfemoral amputees: a case description. Med Boil. Eng. 2015, 54, 1579-1589. [CrossRef]

85. Fernie, G.; Holden, J.; Soto, M. Biofeedback training of knee control in the above-knee amputee. Am. J. Phys. Med. 1978, 57, 161-166.

86. Dietrich, C.; Nehrdich, S.; Seifert, S.; Blume, K.R.; Miltner, W.H.R.; Hofmann, G.O.; Weiss, T. Leg Prosthesis With Somatosensory Feedback Reduces Phantom Limb Pain and Increases Functionality. Front. Neurol. 2018, 9, 270. [CrossRef]

87. Maldonado-Contreras, J.; Marayong, P.; Khoo, I.-H.; Rivera, R.; Ruhe, B.; Wu, W. Proprioceptive improvements of lower-limb amputees under training with a vibrotactile device-A pilot study. In Proceedings of the 2017 IEEE Healthcare Innovations and Point of Care Technologies (HI-POCT), Bethesda, MD, USA, 6-8 November 2017; pp. 229-232.

88. Redd, C.B.; Bamberg, S.J.M. A Wireless Sensory Feedback Device for Real-Time Gait Feedback and Training. IEEE/ASME Trans. Mechatron. 2012, 17, 425-433. [CrossRef] 
89. Davis, I.S. Gait retraining: altering the fingerprint of gait. J. Foot Ankle Res. 2011, 4, A5. [CrossRef]

90. Chow, D.H.; Cheng, C.T. Quantitative analysis of the effects of audio biofeedback on weight-bearing characteristics of persons with transtibial amputation during early prosthetic ambulation. J. Rehabil. Res. Dev. 2000, 37, 255-260.

91. Dingwell, J.B.; Davis, B.L.; Frazder, D.M. Use of an instrumented treadmill for real-time gait symmetry evaluation and feedback in normal and trans-tibial amputee subjects. Prosthet. Orthot. Int. 1996, 20, 101-110. [CrossRef] [PubMed]

92. Clippinger, F.W.; Seaber, A.V.; McElhaney, J.H.; Harrelson, J.M.; Maxwell, G.M. Afferent Sensory Feedback for Lower Extremity Prosthesis. Clin. Orthop. Relat. Res. 1982, 202-206. [CrossRef]

93. Gapsis, J.J.; Grabois, M.; Borrell, R.M.; A Menken, S.; Kelly, M. Limb load monitor: evaluation of a sensory feedback device for controlled weight bearing. Arch. Phys. Med. Rehabil. 1982, 63, 38-41.

94. Zimnicki, B.A.; Fernie, G.R. Biofeedback and the lower extremity amputee: a new training aid. Physiother. Can. 1976, 28, 79-82.

95. Warren, C.G.; Lehmann, J.F. Training procedures and biofeedback methods to achieve controlled partial weight bearing: An assessment. Arch. Phys. Med. Rehabil. 1975, 56, 449-455.

96. Imam, B.; Miller, W.C.; Finlayson, H.C.; Eng, J.J.; Jarus, T. Incidence of lower limb amputation in Canada. Can. J. Public Heal. 2017, 108, 374-380. [CrossRef]

97. Imam, B.; Miller, W.C.; McLaren, L.; Chapman, P.; Finlayson, H. Feasibility of the Nintendo WiiFitTM for improving walking in individuals with a lower limb amputation. SAGE Open Med. 2013, 1, 205031211349794. [CrossRef]

98. Hafner, B.J.; Sawers, A.B. Issues affecting the level of prosthetics research evidence: Secondary analysis of a systematic review. Prosthet. Orthot. Int. 2014, 40, 31-43. [CrossRef]

99. Rommers, G.M.; Vos, L.D.W.; Groothoff, J.W.; Schuiling, C.H.; Eisma, W.H. Epidemiology of lower limb amputees in the north of the Netherlands: Aetiology, discharge destination and prosthetic use. Prosthet. Orthot. Int. 1997, 21, 92-99. [CrossRef]

100. Yilmaz, M.; Gulabi, D.; Kaya, I.; Bayram, E.; Cecen, G.S. The effect of amputation level and age on outcome: an analysis of 135 amputees. Eur. J. Orthop. Surg. Traumatol. 2015, 26, 107-112. [CrossRef] [PubMed]

101. Verhoeff, L.L.; Horlings, C.G.C.; Janssen, L.J.; Bridenbaugh, S.A.; Allum, J.H. Effects of biofeedback on trunk sway during dual tasking in the healthy young and elderly. Gait Posture 2009, 30, 76-81. [CrossRef] [PubMed]

102. Cech, D.J.; Martin, S.T. “Tink” Motor Control and Motor Learning. In Functional Movement Development Across the Life Span; 2012; pp. 68-87.

103. Knaepen, K.; Marusic, U.; Crea, S.; Rodriguez-Guerrero, C.; Vitiello, N.; Pattyn, N.; Mairesse, O.; Lefeber, D.; Meeusen, R. Psychophysiological response to cognitive workload during symmetrical, asymmetrical and dual-task walking. Hum. Mov. Sci. 2015, 40, 248-263. [CrossRef] [PubMed]

104. Czerniecki, J.M.; Morgenroth, D. Metabolic energy expenditure of ambulation in lower extremity amputees: what have we learned and what are the next steps? Disabil. Rehabil. 2015, 39, 143-151. [CrossRef] [PubMed]

105. Moseley, M.J.; Wallace, M.P.; A Stephens, D.; Fielder, A.R.; Smith, L.C.; E Stewart, C. RODS (Randomized Occlusion Dosing Strategies) Study Cooperative Personalized versus standardized dosing strategies for the treatment of childhood amblyopia: study protocol for a randomized controlled trial. Trials 2015, 16, 189. [CrossRef] [PubMed]

106. Highsmith, M.J.; Andrews, C.R.; Millman, C.; Fuller, A.; Kahle, J.T.; Klenow, T.D.; Lewis, K.L.; Bradley, R.C.; Orriola, J. Gait Training Interventions for Lower Extremity Amputees: A Systematic Literature Review. Technol. Innov. 2016, 18, 99-113. [CrossRef]

107. Michaud, S.B.; A Gard, S.; Childress, D.S. A preliminary investigation of pelvic obliquity patterns during gait in persons with transtibial and transfemoral amputation. J. Rehabil. Res. Dev. 2000, 37, 1-10.

108. Boonstra, A.M.; Fidler, V.; Eisma, W.H. Walking speed of normal subjects and amputees: Aspects of validity of gait analysis. Prosthet. Orthot. Int. 1993, 17, 78-82. [CrossRef]

109. Nelson, L.A. The Role of Biofeedback in Stroke Rehabilitation: Past and Future Directions. Top. Stroke Rehabil. 2007, 14, 59-66. [CrossRef]

110. Munin, M.C.; Guzman, M.C.E.-D.; Boninger, M.L.; Fitzgerald, S.G.; E Penrod, L.; Singh, J. Predictive factors for successful early prosthetic ambulation among lower-limb amputees. J. Rehabil. Res. Dev. 2001, 38, 379-384. 
111. Simic, M.; Hinman, R.S.; Wrigley, T.; Bennell, K.L.; Hunt, M.A. Gait modification strategies for altering medial knee joint load: A systematic review. Arthr. Rheum. 2010, 63. [CrossRef] [PubMed]

112. Chamorro-Moriana, G.; Moreno, A.J.; Sevillano, J.L. Technology-Based Feedback and Its Efficacy in Improving Gait Parameters in Patients with Abnormal Gait: A Systematic Review. Sensors 2018, 18, 142. [CrossRef]

113. Bamberg, S.J.M.; Carson, R.J.; Stoddard, G.; Dyer, P.S.; Webster, J.B. The Lower Extremity Ambulation Feedback System for Analysis of Gait Asymmetries: Preliminary Design and Validation Results. JPO J. Prosthet. Orthot. 2010, 22, 31-36. [CrossRef]

114. Liu, C.; De Macedo, L.; Finley, J. Conservation of Reactive Stabilization Strategies in the Presence of Step Length Asymmetries During Walking. Front. Hum. Neurosci. 2018, 212, 251. [CrossRef] [PubMed]

115. Hirokawa, S.; Matsumura, K. Biofeedback gait training system for temporal and distance factors. Med Boil. Eng. 1989, 27, 8-13. [CrossRef] [PubMed]

116. Lee, B.-C.; Fung, A.; Thrasher, T.A. The Effects of Coding Schemes on Vibrotactile Biofeedback for Dynamic Balance Training in Parkinson's Disease and Healthy Elderly Individuals. IEEE Trans. Neural Syst. Rehabil. Eng. 2018, 26, 153-160. [CrossRef]

117. Coffey, L.; O'Keeffe, F.; Gallagher, P.; Desmond, D.; Lombard-Vance, R. Cognitive functioning in persons with lower limb amputations: a review. Disabil. Rehabil. 2012, 34, 1950-1964. [CrossRef]

118. Hamamura, S.; Chin, T.; Kuroda, R.; Akisue, T.; Iguchi, T.; Kohno, H.; Kitagawa, A.; Tsumura, N.; Kurosaka, M. Factors Affecting Prosthetic Rehabilitation Outcomes in Amputees of Age 60 Years and Over. J. Int. Med Res. 2009, 37, 1921-1927. [CrossRef]

119. Andrysek, J.; Klejman, S.; Steinnagel, B.; Torres-Moreno, R.; Zabjek, K.F.; Salbach, N.M.; Moody, K. Preliminary Evaluation of a Commercially Available Videogame System as an Adjunct Therapeutic Intervention for Improving Balance Among Children and Adolescents With Lower Limb Amputations. Arch. Phys. Med. Rehabil. 2012, 93, 358-366. [CrossRef]

120. Pompeu, J.E.; dos Santos Mendes, F.A.; da Silva, K.G.; Lobo, A.M.; de Paula Oliveira, T.; Zomignani, A.P.; Piemonte, M.E.P. Effect of Nintendo WiiTM-based motor and cognitive training on activities of daily living in patients with Parkinson's disease: a randomised clinical trial. Physiotherapy 2012, 98, 196-204. [CrossRef]

(C) 2020 by the authors. Licensee MDPI, Basel, Switzerland. This article is an open access article distributed under the terms and conditions of the Creative Commons Attribution (CC BY) license (http://creativecommons.org/licenses/by/4.0/). 